\title{
ANALYSIS OF A DIFFUSE-INTERFACE MODEL FOR THE BINARY VISCOUS INCOMPRESSIBLE FLUIDS WITH THERMO-INDUCED MARANGONI EFFECTS*
}

\author{
HAO WU ${ }^{\dagger}$ AND XIANG XU‡
}

\begin{abstract}
In this paper we study the well-posedness and long-time dynamics of a diffuseinterface model for the mixture of two viscous incompressible Newtonian fluids with thermo-induced Marangoni effects. The governing system consists of the Navier-Stokes equations coupled with phase-field and energy transport equations. We first derive an energy inequality that illustrates the dissipative nature of the system under the assumption that the initial temperature variation is properly small. Then we establish the existence of weak/strong solutions and discuss the long-time behavior as well as the stability of the system.
\end{abstract}

Key words. Phase-field model, Navier-Stokes equations, Marangoni effects, well-posedness, long-time dynamics.

AMS subject classifications. 35Q35, 35K55, 76D05.

\section{Introduction}

The study of interface dynamics is of great importance in the hydrodynamic theory of complex fluids. In the classical approaches (e.g., the sharp-interface model), the interface is usually considered to be an $n-1$ dimensional free surface of zero width that evolves in time with the fluid. The resulting hydrodynamic system describing the mixture of two immiscible Newtonian fluids with a free interface usually consists of Navier-Stokes equations in each fluid domain with kinematic and force balance boundary conditions on the interface. On the other hand, the so-called diffuse-interface model (or phase-field model), recognizes micro-scale mixing of the macroscopically immiscible fluids and the interface represents a thin region with a steep transition property between two fluids (cf. Anderson et al. [2]). Within this region, the fluid is mixed and has to store certain "mixing energy". The diffuse-interface model can be viewed as a physically motivated level-set method that describes the interface by a proper mixing energy. Compared with the sharp-interface model, the diffuse-interface model can describe topological transitions of interfaces (like pinchoff and reconnection) in a natural way (cf. Lowengrub et al. [21]) and it has many advantages in numerical simulations of the interfacial motion (cf. $[8,18,33]$ and references therein).

The Marangoni effect was initially observed by Thomson [30] during the study of the interesting phenomenon "tears of wine". Afterwards this phenomenon was defined in more detail in Marangoni [22] in terms of surface tension gradients and named after the author. The Marangoni effect is a phenomenon whereby mass transfer occurs due to differences in surface tension. Such differences can either be attributed

${ }^{*}$ Received: April 17, 2012; accepted (in revised form): July 20, 2012. Communicated by John Lowengrub.

H. Wu was partially supported by NSF of China 11001058, SRFDP, and the Fundamental Research Funds for the Central Universities. X. Xu was partially supported by NSF grant DMS-0806703.

†School of Mathematical Sciences and Shanghai Key Laboratory for Contemporary Applied Mathematics, Fudan University, 200433 Shanghai, China (haowufd@yahoo.com).

${ }^{\ddagger}$ Department of Mathematical Sciences, Carnegie Mellon University, Pittsburgh, PA 15213, USA (xuxiang@andrew.cmu.edu). 
to non-uniform distributions of surfactants (cf. Mendes-Tatsis and Agble [23]) or to the existence of temperature gradient in the neighborhood of the interface (cf. Sterling and Scriven [26]). The latter is called the thermo-capillary convection or the Marangoni-Benard convection, which becomes more and more important in the application of complex fluids, liquid-gas systems, and ocean-geophysical dynamics (cf. e.g. $[3,4,15,16,34])$.

The conventional Marangoni-Benard convection for the mixture of two Newtonian flows can be described by a sharp-interface model involving the Boussinesq approximation (cf. e.g., Liu et al. [19]):

$$
\begin{aligned}
& \rho\left(u_{t}+(u \cdot \nabla) u\right)+\nabla p-\nu \Delta u=-\rho_{\theta} g \mathbf{j}, \\
& \nabla \cdot u=0, \\
& \theta_{t}+u \cdot \nabla \theta=k \Delta \theta
\end{aligned}
$$

where $u, p$, and $\theta$ stand for the fluid velocity, the pressure, and the relative temperature (with respect to the reference background temperature $\theta_{b}$, which is assumed to be a constant for the sake of simplicity), respectively. $\rho$ is the density of fluid mixture, $\nu$ is the viscosity, $g$ is the gravitational acceleration, $\mathbf{j}$ is the upward direction and $k>0$ is the thermal diffusion constant. We assume that the temperature-dependent density $\rho_{\theta}$ is described by the Boussinesq approximation

$$
\rho_{\theta}=\rho(1-\alpha \theta),
$$

where $\alpha$ is the coefficient of thermal expansion. The background density $\rho$ is assumed to be a constant and the difference between the actual density and $\rho$ only contributes to the buoyancy force. Interface conditions are given by

$$
\begin{aligned}
& l_{t}+u \cdot \nabla l=0, \\
& {[T] \cdot \mathbf{n}=-\sigma H \mathbf{n}+(\tau \cdot \nabla \sigma) \tau,}
\end{aligned}
$$

where $l$ stands for the interface length of the mixture. The kinematic condition (1.5) indicates that the surface $(l=0)$ evolves with the fluid. Equation (1.6) is the balance of forces on the interface, where $H$ is the curvature of the interface, $[T]$ is the jump of the stress across the interface, $\sigma$ is the surface tension, $\tau$ is the tangential direction on the interface and $\mathbf{n}$ is the normal direction.

In this paper, we shall investigate a diffuse-interface model, which was used to describe the thermo-induced Marangoni effects in the mixture of two incompressible Newtonian fluids. A phase-field variable $\phi$ is introduced as the volume fraction to demarcate the two species and to indicate the location of the interface. The region $\{x: \phi(x, t)=1\}$ is occupied by fluid 1 while $\{x: \phi(x, t)=-1\}$ is occupied by fluid 2 . The interface is represented by $\{x: \phi(x, t)=0\}$, with a (fixed) transition layer of thickness $\varepsilon$. In the diffuse-interface approach, one usually introduces an elastic (mixing) energy of Ginzburg-Landau type

$$
E(\phi)=\int_{\Omega}\left[\frac{1}{2}|\nabla \phi|^{2}+F(\phi)\right] d x
$$

which represents the competition between the hydrophobic and hydrophilic effects of the two different species. The physically relevant energy density function $F$ that 
represents the two phases of the mixture usually has a double-well structure. A typical example of $F$ is the so-called logarithmic potential (cf. Cahn and Hillard [6])

$$
F(\phi)=\gamma_{1}\left(1-\phi^{2}\right)+\gamma_{2}[(1+\phi) \ln (1+\phi)+(1-\phi) \ln (1-\phi)], \quad \gamma_{1}, \gamma_{2}>0 .
$$

In practice, this (singular) potential is often replaced by a smooth double-well polynomial approximation

$$
F(\phi)=\frac{1}{4 \varepsilon^{2}}\left(\phi^{2}-1\right)^{2} .
$$

In this paper, we start with the simple case that the two components of the binary fluid have matched densities and the same constant viscosity $\nu$ as well as the same constant heat conductivity $k$. As a consequence, we consider the following system (cf. e.g., $[18,19,27])$ :

$$
\begin{aligned}
\rho\left(u_{t}+(u \cdot \nabla) u\right)+\nabla p-\nu \Delta u & =-\nabla \cdot\left[\lambda(\theta) \nabla \phi \otimes \nabla \phi-\lambda(\theta)\left(\frac{1}{2}|\nabla \phi|^{2}+F(\phi)\right) \mathbb{I}\right]-\rho_{\theta} g \mathbf{j}, \\
\nabla \cdot u & =0 \\
\phi_{t}+u \cdot \nabla \phi & =\gamma\left(\Delta \phi-F^{\prime}(\phi)\right), \\
\theta_{t}+u \cdot \nabla \theta & =k \Delta \theta,
\end{aligned}
$$

for $(x, t) \in \Omega \times(0,+\infty)$. Here, we assume that $\Omega$ is a bounded domain in $\mathbb{R}^{n}(n=2,3)$ with smooth boundary $\Gamma$. The usual Kronecker product is denoted by $\otimes$, i.e., $(a \otimes b)_{i j}=a_{i} b_{j}$ for $a, b \in \mathbb{R}^{n}$. The system (1.8)-(1.11) contains the Navier-Stokes equations, an Allen-Cahn type equation for the phase-field function, and an energy transport equation for the temperature. The parameter $\gamma$ represents the microscopic elastic relaxation time due to the presence of the microstructure of the mixture. As $\gamma \rightarrow 0$, the internal dissipative mechanism will disappear and the limiting equation is equivalent to the mass transport equation for incompressible fluids (cf. $[19,34]$ ). The temperature-dependent surface tension coefficient $\lambda(\theta)$ is supposed to be

$$
\lambda(\theta)=\lambda_{0}(a-b \theta),
$$

where $\lambda_{0}>0, a>0, b \neq 0$ are constants. Usually $\lambda_{0}$ is assumed to be proportional to the interface length $\varepsilon$ (cf. Sun et al. [27]). Numerical experiments have been made in the recent paper Sun et al. [27] to illustrate the role played by thermal energy in the interfacial dynamics of two-phase flows due to the thermo-induced surface tension heterogeneity on the interface. Their results suggest that the system (1.8)-(1.11) (and its generalizations) turns out to be a suitable mathematical representation that reflects the thermo-induced Marangoni effects in the mixture of fluids.

We suppose that the system (1.8)-(1.11) is subject to the initial conditions

$$
\left.u\right|_{t=0}=u_{0}(x) \text { with } \nabla \cdot u_{0}=0,\left.\quad \phi\right|_{t=0}=\phi_{0}(x),\left.\quad \theta\right|_{t=0}=\theta_{0}(x), \quad x \in \Omega .
$$

Moreover, we assume a no-slip boundary condition on the velocity $u$,

$$
u(x, t)=0, \quad(x, t) \in \Gamma \times(0,+\infty),
$$


nonhomogeneous Dirichlet boundary condition on the phase function $\phi$,

$$
\phi(x, t)=-1, \quad(x, t) \in \Gamma \times(0,+\infty),
$$

and homogeneous Dirichlet boundary condition on the temperature $\theta$,

$$
\theta(x, t)=0, \quad(x, t) \in \Gamma \times(0,+\infty) .
$$

The goal of this paper is to provide a detailed mathematical theory of existence, uniqueness, regularity, and long-time behavior of solutions to the non-isothermal Navier-Stokes-Allen-Cahn system (1.8)-(1.15). First, we prove the existence of global weak solutions in two and three spatial dimensions (cf. Theorem 2.2). Next, we obtain the existence and uniqueness of a global strong solution in $2 D$ (cf. Theorem 3.2), a local strong solution in $3 D$ (cf. Theorem 3.3), and a global strong solutions in $3 D$ provided that the viscosity $\nu$ is properly large (cf. Theorem 3.4). The long-time dynamics of the system seems to be more complicated than the uncoupled Navier-Stokes equations. We prove that as $t \rightarrow+\infty$, the phase function $\phi$ converges to a solution of the stationary Allen-Cahn equation and the velocity $u$ as well as the temperature $\theta$ converges to zero (cf. Theorem 4.3). Stability for minimizers of the elastic energy is also discussed (cf. Theorem 4.9). We just remark that our results can be easily extended to the cases with more general Dirichlet boundary conditions for the phase function. For instance, (1.14) can be replaced by $\left.\phi(x, t)\right|_{\Gamma}=h(x)$ on $\Gamma \times(0,+\infty)$, with $h(x)=\left.\phi_{0}(x)\right|_{\Gamma}$ and $h(x) \in H^{\frac{3}{2}}(\Gamma),|h(x)| \leq 1$ (we refer to Lin and Liu [17] for a similar situation for a simplified nematic liquid crystal system).

It is easy to verify that for the isothermal case of system (1.8)-(1.15) without the Boussinesq approximation term, there is a dissipative energy law

$$
\begin{aligned}
& \frac{d}{d t}\left(\frac{1}{2}\|u\|^{2}+\frac{\lambda}{2}\|\nabla \phi\|^{2}+\lambda \int_{\Omega} F(\phi) d x\right) \\
= & -\nu \int_{\Omega}|\nabla u|^{2} d x-\lambda \gamma \int_{\Omega}\left|-\Delta \phi+F^{\prime}(\phi)\right|^{2} d x .
\end{aligned}
$$

This basic energy law reveals the underlying physics for the isothermal Navier-StokesAllen-Cahn system and it plays an important role in the study of well-posedness as well as long-time behavior of the system. We refer to the recent work $[9,11]$ for detailed mathematical analysis on an isothermal NSAC system for the incompressible two-phase flows, where long-time behavior of global solutions was analyzed within the theory of infinite-dimensional dissipative dynamical systems (e.g., the existence of global attractors, exponential attractors, trajectory attractors, and convergence to single equiblibria).

However, in our present case, the surface tension parameter $\lambda$ in (1.8) depends on the temperature such that it is no longer a constant. Moreover, the Boussinesq approximation is also applied. These bring us challenges in mathematical analysis of the system. We are not able to derive the same dissipative energy equality as for the isothermal case. In particular, the special cancellation between the induced stress term in the momentum equation and the transport term in the phase-field equation is no longer valid (see Remark 2.2 below). This relation is crucial to derive the dissipative energy law like $(1.16)$ (cf. $[9,17,33]$ ). Nevertheless, taking advantage of proper maximum principles for the phase function and temperature, we show that 
if the initial temperature variation is not large (bounded in terms of coefficients of the system), we can derive an energy inequality for the system (1.8)-(1.15), which reflects the dissipative nature of the flow.

We remark that in the phase-field equation (1.10), the dynamics of the phase function $\phi$ is assumed to be driven by a gradient flow of Allen-Cahn type. In order to keep the conservation of overall volume fraction, people usually assume that the internal dissipation is described through a Cahn-Hillard equation (with convection) for $\phi$, which can be viewed as a gradient flow of the elastic energy in the Sobolev space $H^{-1}$ (cf. $[18,21]$ ). The resulting system is nevertheless much more involved in mathematical analysis, because it contains a fourth-order differential operator and thus the maximum principle for the phase function $\phi$ no longer holds (see, for instance, $[1,5,10,35]$ for the isothermal Navier-Stokes-Cahn-Hilliard system). It seems that our results cannot be extended to this case in a straightforward way. For instance, in the derivation of the dissipative energy inequality (2.3), we rely on the $L^{\infty}$-estimate of $\phi$, which will no longer be available due to the lack of maximum principle. This problem will be studied in our future work.

The rest of the paper is organized as follows. In Section 2, we derive an energy inequality that guarantees the dissipative nature of the system and establish the existence of global weak solutions. In Section 3, we discuss existence and uniqueness of strong solutions in both $2 D$ and $3 D$. In Section 4 , we study the long-time dynamics and stability of the system.

\section{Global weak solutions}

If $X$ is a real Hilbert space with inner product $(\cdot, \cdot)_{X}$, then we denote the induced norm by $\|\cdot\|_{X} \cdot X^{\prime}$ indicates the dual space of $X$ and $\langle\cdot, \cdot\rangle_{X^{\prime}, X}$ will denote the corresponding dual product. We indicate by $\mathbf{X}$ the vectorial space $X^{n}$ endowed with the product structure. For simplicity, the scalar product in $L^{2}(\Omega)$ (also $\mathbf{L}^{2}(\Omega)$ ) will be denoted by $(\cdot, \cdot)$, and the associated norm by $\|\cdot\|$. For two $n \times n$ matrices $M_{1}, M_{2}$, we denote $M_{1}: M_{2}=\operatorname{trace}\left(M_{1} M_{2}^{T}\right)$. Let

$$
\mathcal{V}=C_{0}^{\infty}\left(\Omega, \mathbb{R}^{n}\right) \cap\{v: \nabla \cdot v=0\} .
$$

We denote by $\mathbf{H}$ (respectively $\mathbf{V}$ ) the closure of $\mathcal{V}$ in $\mathbf{L}^{2}$ (respectively $\mathbf{H}^{1}$ ):

$$
\mathbf{H}=\left\{u \in \mathbf{L}^{2}: \nabla \cdot u=0, u \cdot \mathbf{n}=0 \text { on } \Gamma\right\}, \quad \mathbf{V}=\left\{u \in \mathbf{H}_{0}^{1}: \nabla \cdot u=0\right\} .
$$

$\mathbf{H}$ and $\mathbf{V}$ are Hilbert spaces with norms $\|\cdot\|$ and $\|\cdot\|_{\mathbf{H}^{1}}$, respectively. We recall the Stokes operator $S: \mathbf{H}^{2}(\Omega) \cap \mathbf{V} \rightarrow \mathbf{H}$ such that $S u=-\Delta u+\nabla \pi \in \mathbf{H}$, for all $u \in \mathbf{H}^{2}(\Omega) \cap$ V. $S^{-1}$ is a compact linear operator on $\mathbf{H}$ and $\|S \cdot\|$ is a norm on $D(S)$ that is equivalent to the $\mathbf{H}^{2}$-norm. Then there exists a positive constant $C=C(n, \Omega)$, for which (cf. Temam [29])

$$
\|u\|_{\mathbf{H}^{2}}+\|\pi\|_{H^{1} \backslash \mathbb{R}} \leq C\|S u\| .
$$

In the following text, we denote by $C, C_{i}$ the generic constants depending on $a, b$, $g, k, \lambda_{0}, \alpha, \gamma, \Omega, \varepsilon$, and the initial data. Special dependence will be pointed out explicitly in the text if necessary.

Without loss of generality, we assume $\rho=1$ in the remaining part of this paper. Now we introduce the weak formulation of the initial boundary value problem (1.8)(1.15): 
Definition 2.1. For any $T \in(0,+\infty)$, the triple $(u, \phi, \theta)$ satisfying

$$
\begin{aligned}
& u \in L^{\infty}(0, T ; \mathbf{H}) \cap L^{2}(0, T ; \mathbf{V}), \\
& \phi \in L^{\infty}\left(0, T ; H^{1} \cap L^{\infty}\right) \cap L^{2}\left(0, T ; H^{2}\right), \quad|\phi| \leq 1, \text { a.e. in } \Omega \times[0, T], \\
& \theta \in L^{\infty}\left(0, T ; H_{0}^{1} \cap L^{\infty}\right) \cap L^{2}\left(0, T ; H^{2}\right),
\end{aligned}
$$

is called a weak solution of the problem (1.8)-(1.11) if the initial and boundary conditions (1.12)-(1.15) are satisfied and for a.e. $t \in(0, T)$,

$$
\begin{aligned}
& \left\langle u_{t}, v\right\rangle_{\mathbf{V}^{\prime}, \mathbf{V}}+\int_{\Omega}(u \cdot \nabla) u \cdot v d x+\nu \int_{\Omega} \nabla u: \nabla v d x \\
= & \int_{\Omega}[\lambda(\theta) \nabla \phi \otimes \nabla \phi]: \nabla v d x+\alpha g \int_{\Omega} \theta \mathbf{j} \cdot v d x, \quad \forall v \in \mathbf{V}, \\
& \phi_{t}+u \cdot \nabla \phi=\gamma\left(\Delta \phi-F^{\prime}(\phi)\right), \quad \text { a.e. in } \Omega, \\
& \theta_{t}+u \cdot \nabla \theta=k \Delta \theta, \quad \text { a.e. in } \Omega, \\
& \left.u\right|_{t=0}=u_{0}(x),\left.\quad \phi\right|_{t=0}=\phi_{0}(x),\left.\quad \theta\right|_{t=0}=\theta_{0}(x), \quad \text { in } \Omega .
\end{aligned}
$$

REMARK 2.1. In order to derive the variational formulation for $u$, we use the following facts due to the incompressibility condition: for any $v \in \mathbf{V}$, it holds that

$$
\begin{aligned}
& \int_{\Omega} \nabla p \cdot v d x=\int_{\Omega} \mathbf{j} \cdot v d x=0, \\
& \int_{\Omega}\left[\nabla \cdot\left(\lambda(\theta)\left(\frac{1}{2}|\nabla \phi|^{2}+F(\phi)\right) \mathbb{I}\right)\right] \cdot v d x=\int_{\Omega} \nabla\left[\lambda(\theta)\left(\frac{1}{2}|\nabla \phi|^{2}+F(\phi)\right)\right] \cdot v d x=0 .
\end{aligned}
$$

Next, we state the result on the existence of global-in-time weak solutions.

Theorem 2.2 (Existence of weak solutions). Suppose $n=2,3$. For any initial data $\left(u_{0}, \phi_{0}, \theta_{0}\right) \in \mathbf{H} \times\left(H^{1}(\Omega) \cap L^{\infty}(\Omega)\right) \times\left(H_{0}^{1}(\Omega) \cap L^{\infty}(\Omega)\right)$ satisfying

$$
\left\|\phi_{0}\right\|_{L^{\infty}} \leq 1, \quad\left\|\theta_{0}\right\|_{L^{\infty}} \leq \frac{1}{4 C_{1}^{2}|b|} \sqrt{\frac{a \gamma \nu}{2 \lambda_{0}}}
$$

where $C_{1}$ is a constant depending only on $n$ and $\Omega$, the problem (1.8)-(1.15) admits at least one global weak solution such that

$$
\begin{aligned}
& u \in L^{\infty}(0,+\infty ; \mathbf{H}) \cap L_{l o c}^{2}(0,+\infty ; \mathbf{V}), \\
& \phi \in L^{\infty}\left(0,+\infty ; H^{1} \cap L^{\infty}\right) \cap L_{l o c}^{2}\left(0,+\infty ; H^{2}\right), \quad|\phi| \leq 1, \text { a.e. in } \Omega \times[0,+\infty), \\
& \theta \in L^{\infty}\left(0,+\infty ; H_{0}^{1} \cap L^{\infty}\right) \cap L_{l o c}^{2}\left(0,+\infty ; H^{2}\right) .
\end{aligned}
$$

2.1. Dissipative energy inequality. An important feature of problem (1.8)(1.15) is that $\phi$ and $\theta$ satisfy the following weak maximum principles, which will be useful in the derivation of the dissipative energy inequality for the system.

Lemma 2.3. Suppose $u \in L^{\infty}(0, T ; \mathbf{H}) \cap L^{2}(0, T ; \mathbf{V})$. If $\phi \in L^{\infty}\left(0, T ; H^{1} \cap L^{\infty}\right) \cap$ $L^{2}\left(0, T ; H^{2}\right)$ is the weak solution of the initial boundary value problem

$$
\begin{aligned}
& \phi_{t}+u \cdot \nabla \phi=\gamma\left(\Delta \phi-F^{\prime}(\phi)\right), \quad \text { a.e. in } \Omega, \\
& \left.\phi(x, t)\right|_{\Gamma}=-1, \quad(x, t) \in \Gamma \times(0, T),
\end{aligned}
$$




$$
\left.\phi\right|_{t=0}=\phi_{0}(x) \in H^{1} \cap L^{\infty} \text {, with }\left|\phi_{0}\right| \leq 1 \text { a.e. in } \Omega,
$$

then $|\phi(x, t)| \leq 1$, a.e. in $\Omega$ for each $t \in(0, T)$.

Proof. The proof is similar to that for the liquid crystal system (cf. e.g., [7,17]), so we omit the details here.

Lemma 2.4. Suppose $u \in L^{\infty}(0, T ; \mathbf{H}) \cap L^{2}(0, T ; \mathbf{V})$. If $\theta \in L^{\infty}\left(0, T ; H_{0}^{1} \cap L^{\infty}\right) \cap$ $L^{2}\left(0, T ; H^{2}\right)$ is the weak solution of the initial boundary value problem

$$
\begin{aligned}
& \theta_{t}+u \cdot \nabla \theta=k \Delta \theta, \quad \text { a.e. in } \Omega, \\
& \left.\theta\right|_{\Gamma}=0, \quad(x, t) \in \Gamma \times(0, T), \\
& \left.\theta\right|_{t=0}=\theta_{0}(x) \in H_{0}^{1} \cap L^{\infty},
\end{aligned}
$$

then $\|\theta(t)\|_{L^{\infty}} \leq\left\|\theta_{0}\right\|_{L^{\infty}}$ for every $t \in(0, T)$.

Proof. Multiplying the equation by $|\theta|^{q-1} \theta(q>1)$, integrating over $\Omega$, we get

$$
\frac{1}{1+q} \frac{d}{d t} \int_{\Omega}|\theta|^{1+q} d x+\frac{1}{1+q} \int_{\Omega} u \cdot \nabla|\theta|^{q+1} d x+\frac{4(q-1)}{(1+q)^{2}} \int_{\Omega} k\left|\nabla\left(|\theta|^{\frac{q-1}{2}} \theta\right)\right|^{2} d x=0,
$$

which implies that

$$
\|\theta(t)\|_{L^{q}} \leq\left\|\theta_{0}\right\|_{L^{q}} \leq|\Omega|^{\frac{1}{1+q}}\left\|\theta_{0}\right\|_{L^{\infty}}, \quad \forall q>1, \quad t \in(0, T) .
$$

Taking the limit $q \rightarrow+\infty$, we arrive at our conclusion.

In what follows, we derive a dissipative energy inequality, which turns out to be crucial in the study of well-posedness as well as long-time dynamics of the problem (1.8)-(1.15).

Proposition 2.5 (Dissipative energy inequality). For $n=2,3$, we assume that the initial phase function $\phi_{0}$ and the initial temperature $\theta_{0}$ satisfy the assumption (2.2). Then there exist constants $\zeta, \omega>0$ that depend only on $\Omega$ and coefficients of the system such that if $(u, \phi, \theta)$ is a smooth solution to the problem (1.8)-(1.15), then the following energy inequality holds:

$$
\frac{d \mathcal{E}}{d t} \leq-\frac{\nu}{2}\|\nabla u\|^{2}-a \lambda_{0} \gamma\left\|\Delta \phi-F^{\prime}(\phi)\right\|^{2}-k \zeta\|\Delta \theta\|^{2} \leq 0, \quad \forall t>0,
$$

where

$$
\begin{array}{cc}
\mathcal{E}(t) & =\|u(t)\|^{2}+a \lambda_{0}\|\nabla \phi(t)\|^{2}+2 a \lambda_{0} \int_{\Omega} F(\phi(t)) d x \\
& +\zeta\|\nabla \theta(t)\|^{2}+\omega\|\theta(t)\|^{2} \\
\geq 0 . &
\end{array}
$$

Proof. Multiplying (1.8) with $u$, (1.10) with $-a \lambda_{0}\left(\Delta \phi-F^{\prime}(\phi)\right)$, (1.11) with $-\zeta \Delta \theta$ ( $\zeta>0$ is a constant to be determined later), respectively, adding them up, and integrating over $\Omega$, we have

$$
\frac{1}{2} \frac{d}{d t}\left(\|u\|^{2}+a \lambda_{0}\|\nabla \phi\|^{2}+2 a \lambda_{0} \int_{\Omega} F(\phi) d x+\zeta\|\nabla \theta\|^{2}\right)
$$


610

$$
\begin{aligned}
& \quad+\nu\|\nabla u\|^{2}+a \gamma \lambda_{0}\left\|\Delta \phi-F^{\prime}(\phi)\right\|^{2}+k \zeta\|\Delta \theta\|^{2} \\
& =\int_{\Omega}(\lambda(\theta) \nabla \phi \otimes \nabla \phi): \nabla u d x+\alpha \int_{\Omega} \theta g \mathbf{j} \cdot u d x+\zeta \int_{\Omega}(u \cdot \nabla) \theta \Delta \theta d x \\
& \quad+a \lambda_{0} \int_{\Omega}(u \cdot \nabla) \phi\left(\Delta \phi-F^{\prime}(\phi)\right) d x \\
& :=J_{1}+J_{2}+J_{3}+J_{4} .
\end{aligned}
$$

In the following we just treat the case $n=3$, while the case $n=2$ is similar. Recall the Gagliardo-Nirenberg inequality

$$
\|\nabla \phi\|_{\mathbf{L}^{4}} \leq C_{1}\left(\|\Delta \phi\|^{\frac{1}{2}}\|\phi\|_{L^{\infty}}^{\frac{1}{2}}+\|\phi\|_{L^{\infty}}\right), \quad \forall \phi \in H^{2},
$$

where $C_{1}=C_{1}(n, \Omega)$ depends only on $n$ and $\Omega$. Combining it with the Poincaré inequality and the Young inequality, we deduce that

$$
\begin{aligned}
J_{1}+J_{4} & =-a \lambda_{0} \int_{\Omega} u \cdot \nabla\left(\frac{|\nabla \phi|^{2}}{2}+F(\phi)\right) d x-b \lambda_{0} \int_{\Omega} \theta \nabla u:(\nabla \phi \otimes \nabla \phi) d x \\
& \leq|b| \lambda_{0}\|\theta\|_{L^{\infty}}\|\nabla u\|\|\nabla \phi\|_{\mathbf{L}^{4}}^{2} \\
& \leq \frac{\nu}{4}\|\nabla u\|^{2}+\frac{|b|^{2} \lambda_{0}^{2}}{\nu}\|\theta\|_{L^{\infty}}^{2}\|\nabla \phi\|_{\mathbf{L}^{4}}^{4} \\
& \leq \frac{\nu}{4}\|\nabla u\|^{2}+\frac{8 C_{1}^{4}|b|^{2} \lambda_{0}^{2}}{\nu}\|\theta\|_{L^{\infty}}^{2}\|\Delta \phi\|^{2}\|\phi\|_{L^{\infty}}^{2}+\frac{8 C_{1}^{4}|b|^{2} \lambda_{0}^{2}}{\nu}\|\theta\|_{L^{\infty}}^{2} \\
\leq & \frac{\nu}{4}\|\nabla u\|^{2}+\frac{16 C_{1}^{4}|b|^{2} \lambda_{0}^{2}}{\nu}\|\theta\|_{L^{\infty}}^{2}\left(\left\|\Delta \phi-F^{\prime}(\phi)\right\|^{2}+\left\|F^{\prime}(\phi)\right\|^{2}\right)\|\phi\|_{L^{\infty}}^{2} \\
& \quad+\frac{8 C_{1}^{4}|b|^{2} \lambda_{0}^{2}}{\nu}\|\theta\|_{L^{\infty}}^{2} .
\end{aligned}
$$

Then by Lemmas $2.3,2.4$, and the assumption (2.2), we obtain that

$$
\begin{aligned}
& \frac{16 C_{1}^{4}|b|^{2} \lambda_{0}^{2}}{\nu}\|\theta\|_{L^{\infty}}^{2}\left\|\Delta \phi-F^{\prime}(\phi)\right\|^{2}\|\phi\|_{L^{\infty}}^{2} \leq \frac{a \lambda_{0} \gamma}{2}\left\|\Delta \phi-F^{\prime}(\phi)\right\|^{2}, \\
& \frac{16 C_{1}^{4}|b|^{2} \lambda_{0}^{2}}{\nu}\|\theta\|_{L^{\infty}}^{2}\left\|F^{\prime}(\phi)\right\|^{2}\|\phi\|_{L^{\infty}}^{2} \leq \frac{16 C_{1}^{4} C_{2}|b|^{2} \lambda_{0}^{2}|\Omega|^{2}}{\nu \varepsilon^{4}}\|\Delta \theta\|^{\frac{3}{2}}\|\theta\|^{\frac{1}{2}} \\
& \leq \frac{k \zeta}{8}\|\Delta \theta\|^{2}+\frac{54 \cdot 16^{4} C_{1}^{16} C_{2}^{4}|b|^{8} \lambda_{0}^{8}|\Omega|^{8}}{\nu^{4} \varepsilon^{16} k^{3} \zeta^{3}}\|\theta\|^{2},
\end{aligned}
$$

and

$$
\begin{aligned}
\frac{8 C_{1}^{4}|b|^{2} \lambda_{0}^{2}}{\nu}\|\theta\|_{L^{\infty}}^{2} & \leq \frac{8 C_{1}^{4} C_{2}|b|^{2} \lambda_{0}^{2}}{\nu}\|\Delta \theta\|^{\frac{3}{2}}\|\theta\|^{\frac{1}{2}} \\
& \leq \frac{k \zeta}{8}\|\Delta \theta\|^{2}+\frac{54 \cdot 8^{4} C_{1}^{16} C_{2}^{4}|b|^{8} \lambda_{0}^{8}}{\nu^{4} k^{3} \zeta^{3}}\|\theta\|^{2},
\end{aligned}
$$

where $C_{2}$ depends only on $\Omega$. As a result,

$$
J_{1}+J_{4} \leq \frac{\nu}{4}\|\nabla u\|^{2}+\frac{a \lambda_{0} \gamma}{2}\left\|\Delta \phi-F^{\prime}(\phi)\right\|^{2}+\frac{k \zeta}{4}\|\Delta \theta\|^{2}+\frac{C_{3}}{\zeta^{3}}\|\theta\|^{2},
$$

with

$$
C_{3}=\frac{54 \cdot 16^{4} C_{1}^{16} C_{2}^{4}|b|^{8} \lambda_{0}^{8}|\Omega|^{8}+54 \cdot 8^{4} \varepsilon^{16} C_{1}^{16} C_{2}^{4}|b|^{8} \lambda_{0}^{8}}{\nu^{4} \varepsilon^{16} k^{3}} .
$$


Next, by the Poincaré inequality

$$
J_{2} \leq|\alpha||g|\|\theta\|\|\| u\left\|\leq C_{P}|\alpha|\right\| g \mid\|\theta\|\|\nabla u\| \leq \frac{\nu}{4}\|\nabla u\|^{2}+\frac{C_{P}^{2}|\alpha|^{2}|g|^{2}}{\nu}\|\theta\|^{2},
$$

where $C_{P}$ depends only on $\Omega$. For $J_{3}$, we have

$$
\begin{aligned}
J_{3} & =-\zeta \int_{\Omega} u \cdot \nabla\left(\frac{|\nabla \theta|^{2}}{2}\right) d x+\zeta \int_{\Omega} u \cdot[\nabla \cdot(\nabla \theta \otimes \nabla \theta)] d x \\
& =-\zeta \int_{\Omega} \nabla u:(\nabla \theta \otimes \nabla \theta) d x \\
& \leq \zeta\|\nabla u\|\|\nabla \theta\|_{\mathbf{L}^{4}}^{2} \leq C_{1}^{2} \zeta\|\nabla u\|\|\Delta \theta\|\|\theta\|_{L^{\infty}} \\
& \leq \frac{k \zeta}{4}\|\Delta \theta\|^{2}+\frac{C_{1}^{4} \zeta}{k}\left\|\theta_{0}\right\|_{L^{\infty}}^{2}\|\nabla u\|^{2} \\
& \leq \frac{k \zeta}{4}\|\Delta \theta\|^{2}+\frac{a \gamma \nu \zeta}{4 k|b|^{2} \lambda_{0}}\|\nabla u\|^{2} .
\end{aligned}
$$

Taking

$$
\zeta=\frac{k|b|^{2} \lambda_{0}}{a \gamma}
$$

we infer from the above estimates that

$$
\begin{aligned}
& \frac{d}{d t}\left(\|u\|^{2}+a \lambda_{0}\|\nabla \phi\|^{2}+2 a \lambda_{0} \int_{\Omega} F(\phi) d x+\zeta\|\nabla \theta\|^{2}\right) \\
& +\frac{\nu}{2}\|\nabla u\|^{2}+\gamma a \lambda_{0}\left\|\Delta \phi-F^{\prime}(\phi)\right\|^{2}+k \zeta\|\Delta \theta\|^{2} \\
\leq & C_{4}\|\theta\|^{2},
\end{aligned}
$$

where

$$
C_{4}=\frac{2 C_{3}}{\zeta^{3}}+\frac{2 C_{P}^{2}|\alpha|^{2}|g|^{2}}{\nu}
$$

Multiplying (1.11) by $2 \omega \theta, \omega=\frac{C_{P}^{2} C_{4}}{2 k}>0$, integrating over $\Omega$, and using the Poincaré inequality, we obtain

$$
\omega \frac{d}{d t}\|\theta\|^{2}=-2 \omega k\|\nabla \theta\|^{2} \leq-\frac{2 \omega k\|\theta\|^{2}}{C_{P}^{2}}=-C_{4}\|\theta\|^{2} .
$$

Adding (2.5) with (2.6), we arrive at our conclusion.

REMARK 2.2. For the isothermal case of the system (1.8)-(1.15) without the Boussinesq approximation term, there is a special cancellation between the induced stress term in the Navier-Stokes equations and the convection term in the Allen-Cahn equation, which yields the dissipative energy equality (1.16). However, for the current non-isothermal system (1.8)-(1.15), there exists an extra high-order term

$$
-b \lambda_{0} \int_{\Omega} \theta \nabla u:(\nabla \phi \otimes \nabla \phi) d x
$$


containing the velocity, phase function, and temperature that cannot be eliminated (the Boussinesq approximation is a lower-order term and is easier to handle). In order to overcome this difficulty, we introduce the smallness assumption (2.2) and try to seek certain energy dissipative inequality instead.

REMARK 2.3. It is worth mentioning that the conditions in (2.2) do not involve the interfacial parameter $\varepsilon$.

2.2. Proof of Theorem 2.2. The proof is based on a semi-Galerkin method (see, for instance, Lin and Liu [17] for a simplified nematic liquid crystal system). Let $\left\{w_{i}\right\}(i \in \mathbb{N})$ be an orthonormal basis of $\mathbf{V}$ formed by the eigenvectors of the Stokes problem

$$
-\Delta w_{i}+\nabla P_{i}=\lambda_{i} w_{i}, \quad \text { in } \Omega,\left.\quad w_{i}\right|_{\Gamma}=0,
$$

with $\left\|w_{i}\right\|=1$ and $0<\lambda_{1} \leq \lambda_{2} \leq \cdots \leq \lambda_{n} \leq \cdots$ with $\lambda_{n} \rightarrow \infty$ as $n \rightarrow+\infty$.

For every $m \in \mathbb{N}$, let $\mathbf{V}_{m}=\operatorname{span}\left\{w_{1}, w_{2}, \cdots, w_{m}\right\}$. We denote by $\mathrm{P}_{m}: \mathbf{H} \rightarrow \mathbf{V}_{m}$ the orthogonal projection. Given $\left(u_{0}, \phi_{0}, \theta_{0}\right) \in \mathbf{H} \times\left(H^{1}(\Omega) \cap L^{\infty}(\Omega)\right) \times\left(H_{0}^{1}(\Omega) \cap L^{\infty}(\Omega)\right)$ satisfying (2.2), we consider the following approximate problem:

$$
\begin{aligned}
& \left\langle\partial_{t} u_{m}, v_{m}\right\rangle_{\mathbf{V}^{\prime}, \mathbf{V}}+\int_{\Omega}\left(u_{m} \cdot \nabla\right) u_{m} \cdot v_{m} d x+\nu \int_{\Omega} \nabla u_{m}: \nabla v_{m} d x \\
= & \int_{\Omega}\left[\lambda\left(\theta_{m}\right) \nabla \phi_{m} \otimes \nabla \phi_{m}\right]: \nabla v_{m} d x+\alpha g \int_{\Omega} \theta_{m} \mathbf{j} \cdot v_{m} d x, \quad \forall v_{m} \in \mathbf{V}_{m}, \\
& \partial_{t} \phi_{m}+u_{m} \cdot \nabla \phi_{m}=\gamma\left(\Delta \phi_{m}-F^{\prime}\left(\phi_{m}\right)\right), \quad \text { a.e. in } \Omega, \\
& \partial_{t} \theta_{m}+u_{m} \cdot \nabla \theta_{m}=k \Delta \theta_{m}, \quad \text { a.e. in } \Omega, \\
& \phi_{m}(x, t)=-1, \quad \theta_{m}(x, t)=0 \text { on } \Gamma, \\
& \left.u_{m}\right|_{t=0}=\mathrm{P}_{m} u_{0}(x),\left.\quad \phi_{m}\right|_{t=0}=\phi_{0}(x),\left.\quad \theta_{m}\right|_{t=0}=\theta_{0}(x) .
\end{aligned}
$$

Indeed, we observe that all of the a priori bounds derived (formally) from the energy inequality (2.3) still hold for the approximate problem. If we fix $\tilde{u}_{m} \in C\left([0, T] ; \mathbf{V}_{m}\right)$, then we can find $\phi_{m}=\phi_{m}\left[\tilde{u}_{m}\right]$ and $\theta_{m}=\theta_{m}\left[\tilde{u}_{m}\right]$ solving (2.8) and (2.9) (with $u_{m}=$ $\tilde{u}_{m}$ ), respectively. Inserting $\phi_{m}$ and $\theta_{m}$ into the equation (2.7), we can find a solution $u_{m}=\mathcal{T}\left[\tilde{u}_{m}\right]$ that defines a mapping $\tilde{u}_{m} \mapsto \mathcal{T}\left[\tilde{u}_{m}\right]$. On account of the a priori bounds, we can easily show that $\mathcal{T}$ admits a fixed point by means of the classical Schauder's argument on $\left(0, T_{0}\right)$, with $0<T_{0} \leq T$. Finally, applying again the a priori estimates, we are allowed to conclude that the approximate solutions can be extended to the whole time interval $[0,+\infty)$ (cf. also Ezquerra et al. [7, Appendix]). Since the $a$ priori estimates of the approximate solution are uniform in parameter $m$, using a similar argument as in [17, Section 2] we can pass to the limit $m \rightarrow+\infty$ and complete the proof of Theorem 2.2. The details are omitted here.

Corollary 2.6. Suppose $n=2,3$. Under the assumptions of Theorem 2.2, the weak solution $(u, \phi, \theta)$ to the problem (1.8)-(1.15) satisfies

$$
\|\phi(t)\|_{L^{\infty}} \leq 1, \quad\|\theta(t)\|_{L^{\infty}} \leq \frac{1}{4 C_{1}^{2}|b|} \sqrt{\frac{a \gamma \nu}{2 \lambda_{0}}}, \quad \forall t \geq 0,
$$

and the energy inequality

$$
\mathcal{E}(t)+\int_{0}^{+\infty}\left(\frac{\nu}{2}\|\nabla u\|^{2}+a \lambda_{0} \gamma\left\|\Delta \phi-F^{\prime}(\phi)\right\|^{2}+k \zeta\|\Delta \theta\|^{2}\right) d t \leq \mathcal{E}(0), \quad \forall t \geq 0
$$


which yields the following uniform estimates:

$$
\begin{aligned}
& \|u(t)\|^{2}+\|\phi(t)\|_{H^{1}}^{2}+\|\theta(t)\|_{H^{1}}^{2} \leq M, \quad \forall t \geq 0, \\
& \int_{0}^{+\infty}\left(\|\nabla u(t)\|^{2}+\left\|\Delta \phi(t)-F^{\prime}(\phi(t))\right\|^{2}+\|\Delta \theta(t)\|^{2}\right) d t \leq M,
\end{aligned}
$$

where $M>0$ is a constant depending on $\left\|u_{0}\right\|,\left\|\phi_{0}\right\|_{H^{1}},\left\|\theta_{0}\right\|_{H^{1}}, \Omega$, and coefficients of the system.

\section{Strong solutions}

In this section, we prove the existence and uniqueness of strong solutions to the problem (1.8)-(1.15).

Definition 3.1. For any $T \in(0,+\infty], u_{0} \in \mathbf{V}, \phi_{0} \in H^{2}(\Omega), \theta_{0} \in H^{2}(\Omega) \cap H_{0}^{1}(\Omega)$, we say that the triple $(u, \phi, \theta)$ is a strong solution to the problem (1.8)-(1.15), if $(u, \phi, \theta)$ is a weak solution and

$$
u \in L^{\infty}(0, T ; \mathbf{V}) \cap L^{2}\left(0, T ; \mathbf{H}^{2}\right), \quad \phi, \theta \in L^{\infty}\left(0, T ; H^{2}\right) \cap L^{2}\left(0, T ; H^{3}\right) .
$$

Based on the semi-Galerkin scheme in the previous section, in order to prove the existence of strong solutions, it suffices to derive proper uniform higher-order estimates for the approximate solutions and then pass to the limit $m \rightarrow+\infty$. We observe that the approximate solutions satisfy the same basic energy inequality and higher-order differential inequalities as smooth solutions of the problem (1.8)-(1.15). Thus, for the sake of simplicity, all the calculations below will be carried out formally for smooth solutions.

The main results of this section are as follows.

Theorem 3.2 (Global strong solution in $2 D$ ). Suppose $n=2$. For any $u_{0} \in$ $\mathbf{V}, \phi_{0} \in H^{2}(\Omega), \theta_{0} \in\left(H_{0}^{1}(\Omega) \cap H^{2}(\Omega)\right)$ satisfying the assumption (2.2), the problem (1.8)-(1.15) admits a unique global strong solution such that

$$
\begin{aligned}
& u \in L^{\infty}(0,+\infty ; \mathbf{V}) \cap L_{l o c}^{2}\left(0,+\infty ; \mathbf{H}^{2}\right), \\
& \phi \in L^{\infty}\left(0,+\infty ; H^{2}\right) \cap L_{l o c}^{2}\left(0,+\infty ; H^{3}\right), \\
& \theta \in L^{\infty}\left(0,+\infty ; H^{2} \cap H_{0}^{1}\right) \cap L_{l o c}^{2}\left(0,+\infty ; H^{3}\right) .
\end{aligned}
$$

Theorem 3.3 (Local strong solution in $3 D$ ). Suppose $n=3$. For any $u_{0} \in$ $\mathbf{V}, \phi_{0} \in H^{2}(\Omega), \theta_{0} \in\left(H_{0}^{1}(\Omega) \cap H^{2}(\Omega)\right)$ satisfying the assumption (2.2), the problem (1.8)-(1.15) admits a unique local strong solution.

Theorem 3.4 (Global strong solution in $3 D$ under large viscosity). Suppose $n=3$. For any $u_{0} \in \mathbf{V}, \phi_{0} \in H^{2}(\Omega), \theta_{0} \in\left(H_{0}^{1}(\Omega) \cap H^{2}(\Omega)\right)$ satisfying the assumption (2.2), if in addition, the lower bound of the viscosity, i.e., $\nu$ is sufficiently large (cf. (3.12)), then the problem (1.8)-(1.15) admits a unique global strong solution.

3.1. Two dimensional case. First, we are going to derive a specific type of higher-order energy inequality in the spirit of [17].

Lemma 3.5. Suppose $n=2$. Let $(u, \phi, \theta)$ be a smooth solution to the problem (1.8)(1.15). We introduce the quantity

$$
\mathcal{A}_{1}(t)=\|\nabla u(t)\|^{2}+a \lambda_{0}\left\|\Delta \phi(t)-F^{\prime}(\phi(t))\right\|^{2}+\eta_{1}\|\Delta \theta(t)\|^{2} .
$$


Then the following differential inequality holds:

$$
\begin{aligned}
& \frac{d}{d t} \mathcal{A}_{1}(t)+\nu\|S u\|^{2}+a \lambda_{0} \gamma\left\|\nabla\left(\Delta \phi-F^{\prime}(\phi)\right)\right\|^{2}+\eta_{1} k\|\nabla \Delta \theta\|^{2} \\
\leq & C\left(\mathcal{A}_{1}^{2}(t)+\mathcal{A}_{1}(t)\right), \quad \forall t>0 .
\end{aligned}
$$

$\eta_{1}$ and $C$ are two positive constants which may depend on $\left\|u_{0}\right\|,\left\|\phi_{0}\right\|_{H^{1}},\left\|\theta_{0}\right\|_{H^{1}}, \Omega$, and coefficients of the system.

Proof. We observe that $-\left(\Delta u, u_{t}\right)=\left(S u, u_{t}\right)$, since $u_{t} \in \mathbf{H}$. Besides, due to (1.14), we infer that $\left.\phi_{t}\right|_{\Gamma}=0$, so by (1.13) and the equation (1.10) we see that $\Delta \phi-\left.F^{\prime}(\phi)\right|_{\Gamma}=$ $\phi_{t}+\left.u \cdot \nabla \phi\right|_{\Gamma}=0$. Using equations (1.8) and (1.10), we compute that

$$
\begin{aligned}
& \frac{1}{2} \frac{d}{d t}\left(\|\nabla u\|^{2}+a \lambda_{0}\left\|\Delta \phi-F^{\prime}(\phi)\right\|^{2}\right)+\nu\|S u\|^{2}+a \lambda_{0} \gamma\left\|\nabla\left(\Delta \phi-F^{\prime}(\phi)\right)\right\|^{2} \\
=-(u & \cdot \nabla u, S u)+b \lambda_{0}(\nabla \cdot(\theta \nabla \phi \otimes \nabla \phi), S u)+\alpha g(\theta \mathbf{j}, S u) \\
& -a \lambda_{0}\left(\nabla \phi\left(\Delta \phi-F^{\prime}(\phi)\right), \nabla \pi\right)-a \lambda_{0} \gamma\left(F^{\prime \prime}(\phi)\left(\Delta \phi-F^{\prime}(\phi)\right), \Delta \phi-F^{\prime}(\phi)\right) \\
& \quad-2 a \lambda_{0} \int_{\Omega}\left(\Delta \phi-F^{\prime}(\phi)\right) \nabla_{j} u_{i} \nabla_{j} \nabla_{i} \phi d x
\end{aligned}
$$

In what follows, we proceed to estimate the right-hand side of (3.3) by using the uniform estimates obtained in Corollary 2.6 and properties of the Stokes operator.

$$
\begin{aligned}
K_{1} & \leq \frac{\nu}{32}\|S u\|^{2}+C\|u\|_{\mathbf{L}^{\infty}}^{2}\|\nabla u\|^{2} \\
& \leq \frac{\nu}{32}\|S u\|^{2}+C\|u\|\|\Delta u\|\|\nabla u\|^{2} \\
& \leq \frac{\nu}{16}\|S u\|^{2}+C\|\nabla u\|^{4} .
\end{aligned}
$$

For the second term $K_{2}$, we have

$$
\begin{aligned}
& K_{2}= b \lambda_{0} \int_{\Omega} \nabla_{j} \theta \nabla_{i} \phi \nabla_{j} \phi(S u)_{i} d x+\frac{b \lambda_{0}}{2}\left(\theta \nabla|\nabla \phi|^{2}, S u\right)+b \lambda_{0}(\theta \Delta \phi \nabla \phi, S u) \\
&= b \lambda_{0} \int_{\Omega} \nabla_{j} \theta \nabla_{i} \phi \nabla_{j} \phi(S u)_{i} d x-\frac{b \lambda_{0}}{2}\left(|\nabla \phi|^{2} \nabla \theta, S u\right)+b \lambda_{0}(\theta \Delta \phi \nabla \phi, S u) \\
&:= K_{2 a}+K_{2 b}+K_{2 c} . \\
& K_{2 a}+K_{2 b} \\
& \leq C\|\nabla \theta\|_{\mathbf{L}^{6}}\|\nabla \phi\|_{\mathbf{L}^{6}}^{2}\|S u\| \\
& \leq \frac{\nu}{16}\|S u\|^{2}+C\left(\|\nabla \Delta \theta\|^{\frac{2}{3}}\|\nabla \theta\|^{\frac{4}{3}}+\|\nabla \theta\|^{2}\right)\left(\|\nabla \Delta \phi\|^{\frac{4}{3}}\|\nabla \phi\|^{\frac{8}{3}}+\|\nabla \phi\|^{4}\right) \\
& \leq \frac{\nu}{16}\|S u\|^{2}+C\left(\|\nabla \Delta \theta\|^{\frac{2}{3}}\|\nabla \theta\|^{\frac{4}{3}}+\|\nabla \theta\|^{2}\right) \\
& \times\left(\left\|\nabla\left(\Delta \phi-F^{\prime}(\phi)\right)\right\|^{\frac{4}{3}}+\left\|F^{\prime \prime}(\phi)\right\|_{L^{\infty}}^{\frac{4}{3}}\|\nabla \phi\|^{\frac{4}{3}}+C\right) \\
& \leq \frac{\nu}{16}\|S u\|^{2}+\frac{a \lambda_{0} \gamma}{8}\left\|\nabla\left(\Delta \phi-F^{\prime}(\phi)\right)\right\|^{2}+C_{5}\|\nabla \Delta \theta\|^{2} \\
& \quad+C\left(\|\nabla \theta\|^{2}+\|\nabla \theta\|^{6}\right),
\end{aligned}
$$


where $C_{5}$ is a constant depending on $\left\|u_{0}\right\|,\left\|\phi_{0}\right\|_{H^{1}},\left\|\theta_{0}\right\|_{H^{1}}, \Omega$, and coefficients of the system.

$$
\begin{aligned}
& K_{2 c} \leq \leq b \mid \lambda_{0}\|\theta\|_{L^{\infty}}\|\Delta \phi\|\|\nabla \phi\|_{\mathbf{L}^{\infty}}\|S u\| \\
& \leq C\|\theta\|_{L^{\infty}}\|\Delta \phi\|\|\nabla \phi\|_{\mathbf{H}^{2}}^{2}\|\nabla \phi\|^{\frac{1}{2}}\|S u\| \\
& \leq \frac{\nu}{16}\|S u\|^{2}+C\|\theta\|_{L^{\infty}}^{2}\left(\left\|\Delta \phi-F^{\prime}(\phi)\right\|^{2}+C\right) \\
& \quad \times\left(\left\|\nabla\left(\Delta \phi-F^{\prime}(\phi)\right)\right\|+\left\|\Delta \phi-F^{\prime}(\phi)\right\|+C\right) \\
& \leq \frac{\nu}{16}\|S u\|^{2}+C\left\|\Delta \phi-F^{\prime}(\phi)\right\|^{2}\left\|\nabla\left(\Delta \phi-F^{\prime}(\phi)\right)\right\| \\
& \quad+C\|\theta\|\|\Delta \theta\|\left(\left\|\nabla\left(\Delta \phi-F^{\prime}(\phi)\right)\right\|+\left\|\Delta \phi-F^{\prime}(\phi)\right\|^{3}+C\right) \\
& \leq \frac{\nu}{16}\|S u\|^{2}+\frac{a \lambda_{0} \gamma}{8}\left\|\nabla\left(\Delta \phi-F^{\prime}(\phi)\right)\right\|^{2} \\
& \quad+C\left(\left\|\Delta \phi-F^{\prime}(\phi)\right\|^{2}+\left\|\Delta \phi-F^{\prime}(\phi)\right\|^{4}+\|\Delta \theta\|^{2}+\|\Delta \theta\|^{4}\right) .
\end{aligned}
$$

The remaining terms can be estimated as follows:

$$
\begin{gathered}
K_{3} \leq \mid \alpha\|g\|\|\theta\|\|S u\| \leq \frac{\nu}{16}\|S u\|^{2}+C\|\Delta \theta\|^{2} . \\
K_{4} \leq a \lambda_{0}\|\nabla \pi\|\|\nabla \phi\|_{\mathbf{L}^{4}}\left\|\Delta \phi-F^{\prime}(\phi)\right\|_{L^{4}} \\
\leq C\|S u\|\left(\left\|\Delta \phi-F^{\prime}(\phi)\right\|^{\frac{1}{2}}+C\right)\left\|\Delta \phi-F^{\prime}(\phi)\right\|^{\frac{1}{2}}\left\|\nabla\left(\Delta \phi-F^{\prime}(\phi)\right)\right\|^{\frac{1}{2}} \\
\leq \frac{\nu}{16}\|S u\|^{2}+\frac{a \lambda_{0} \gamma}{8}\left\|\nabla\left(\Delta \phi-F^{\prime}(\phi)\right)\right\|^{2} \\
+C\left(\left\|\Delta \phi-F^{\prime}(\phi)\right\|^{2}+\left\|\Delta \phi-F^{\prime}(\phi)\right\|^{4}\right) . \\
K_{5} \leq a \lambda_{0} \gamma\left\|F^{\prime \prime}(\phi)\right\|_{L^{\infty}}\left\|\Delta \phi-F^{\prime}(\phi)\right\|^{2} \leq C\left\|\Delta \phi-F^{\prime}(\phi)\right\|^{2} . \\
\leq C\left\|\nabla\left(\Delta \phi-F^{\prime}(\phi)\right)\right\|\|\nabla u\|_{\mathbf{L}^{4}}\|\nabla \phi\|_{\mathbf{L}^{4}} \\
\leq \frac{a \lambda_{0} \gamma}{12}\left\|\nabla\left(\Delta \phi-F^{\prime}(\phi)\right)\right\|^{2}+C\|\nabla u\|\|\Delta u\|\left(\left\|\Delta \phi-F^{\prime}(\phi)\right\|+\left\|F^{\prime}(\phi)\right\|\right)\|\nabla \phi\| \\
\leq \frac{\nu}{16}\|S u\|^{2}+\frac{a \lambda_{0} \gamma}{8}\left\|\nabla\left(\Delta \phi-F^{\prime}(\phi)\right)\right\|^{2} \\
+C\left(\|\nabla u\|^{2}+\|\nabla u\|^{4}+\left\|\Delta \phi-F^{\prime}(\phi)\right\|^{4}\right) .
\end{gathered}
$$

It follows from the above estimates and the Sobolev embedding that

$$
\begin{aligned}
& \quad \frac{1}{2} \frac{d}{d t}\left(\|\nabla u\|^{2}+a \lambda_{0}\left\|\Delta \phi-F^{\prime}(\phi)\right\|^{2}\right)+\frac{5 \nu}{8}\|S u\|^{2}+\frac{a \lambda_{0} \gamma}{2}\left\|\nabla\left(\Delta \phi-F^{\prime}(\phi)\right)\right\|^{2} \\
& \leq C_{5}\|\nabla \Delta \theta\|^{2}+C\left(\|\nabla u\|^{4}+\left\|\Delta \phi-F^{\prime}(\phi)\right\|^{4}+\|\Delta \theta\|^{4}\right) \\
& +C\left(\|\Delta u\|^{2}+\left\|\Delta \phi-F^{\prime}(\phi)\right\|^{2}+\|\Delta \theta\|^{2}\right) .
\end{aligned}
$$

We infer from (1.15) that $\left.\theta_{t}\right|_{\Gamma}=0$, so it follows from (1.13) and the $\theta$-equation (1.11) that $\left.\Delta \theta\right|_{\Gamma}=0$. Applying $\Delta$ to both sides of (1.11), and taking the $L^{2}$-inner product of the resultant with $\Delta \theta$, we obtain

$$
\frac{1}{2} \frac{d}{d t}\|\Delta \theta\|^{2}+k\|\nabla \Delta \theta\|^{2}=-\int_{\Omega} \Delta(u \cdot \nabla \theta) \Delta \theta d x:=K_{7},
$$


such that

$$
\begin{aligned}
K_{7} & =\int_{\Omega} \nabla(u \cdot \nabla \theta) \cdot \nabla \Delta \theta d x \\
& \leq \frac{k}{8}\|\nabla \Delta \theta\|^{2}+\frac{2}{k}\|\nabla(u \cdot \nabla \theta)\|^{2} \\
& \leq \frac{k}{8}\|\nabla \Delta \theta\|^{2}+C\left(\|\nabla u\|_{\mathbf{L}^{4}}^{2}\|\nabla \theta\|_{\mathbf{L}^{4}}^{2}+\|u\|_{\mathbf{L}^{\infty}}^{2}\|\Delta \theta\|^{2}\right) \\
& \leq \frac{k}{8}\|\nabla \Delta \theta\|^{2}+C\|\Delta u\|^{\frac{3}{2}}\|u\|^{\frac{1}{2}}\left(\|\Delta \theta\|\|\nabla \theta\|+\|\nabla \theta\|^{2}\right) \\
& \quad+C\|\Delta u\|\|u\|\|\Delta \theta\|^{2} \\
& \leq \frac{k}{4}\|\nabla \Delta \theta\|^{2}+\frac{\nu}{8 k C_{5}}\|S u\|^{2}+C\|\nabla u\|^{2}+C\|\Delta \theta\|^{4} .
\end{aligned}
$$

Hence, multiplying (3.5) by $\eta_{1}=k C_{5}$, and adding the result to (3.4), we deduce our conclusion (3.2) from (3.6). The proof is complete.

\section{Proof of Theorem 3.2.}

Proof. Since $u_{0} \in \mathbf{V}, \phi_{0} \in H^{2}(\Omega), \theta_{0} \in\left(H_{0}^{1}(\Omega) \cap H^{2}(\Omega)\right)$, we have $\mathcal{A}_{1}(0)<+\infty$. It follows from Corollary 2.6 that $\mathcal{A}_{1}(t) \in L^{1}(0,+\infty)$. Then we infer from Lemma 3.5 and [36, Lemma 6.2.1] that $\mathcal{A}_{1}(t)$ is uniformly bounded for all time, which implies $\forall t \geq 0$, it holds that

$$
\begin{aligned}
& \|u(t)\|_{\mathbf{H}^{1}}+\|\phi(t)\|_{H^{2}}+\|\theta(t)\|_{H^{2}} \\
& +\int_{t}^{t+1}\left(\|u(s)\|_{\mathbf{H}^{2}}^{2}+\|\phi(s)\|_{H^{3}}^{2}+\|\theta(s)\|_{H^{3}}^{2}\right) d s \leq C,
\end{aligned}
$$

where $C$ is a positive constant depending on $\left\|u_{0}\right\|_{\mathbf{H}^{1}},\left\|\phi_{0}\right\|_{H^{2}},\left\|\theta_{0}\right\|_{H^{2}}, \Omega$, and coefficients of the system. Then we can prove the existence of a global strong solution, which is actually unique by Proposition 3.9. The proof is complete.

For the weak solution, we still have $\mathcal{A}_{1}(t) \in L^{1}(0,+\infty)$. Then by (3.2) and the uniform Gronwall lemma (cf. Temam [28, Lemma III.1.1]), we conclude that for any $\delta>0$,

$$
\mathcal{A}_{1}(t+\delta) \leq C\left(1+\frac{1}{\delta}\right), \quad \forall t \geq 0
$$

where $C$ is a positive constant depending on $\left\|u_{0}\right\|,\left\|\phi_{0}\right\|_{H^{1}},\left\|\theta_{0}\right\|_{H^{1}}, \Omega$, and coefficients of the system. As a result, we have the following proposition.

Proposition 3.6 (Regularity of weak solutions in $2 D$ ). When $n=2$, under the assumptions of Theorem 2.2, any weak solution to problem (1.8)-(1.15) becomes a strong one for $t>0$ and the following estimate holds:

$$
\|u(t)\|_{\mathbf{H}^{1}}^{2}+\|\phi(t)\|_{H^{2}}^{2}+\|\theta(t)\|_{H^{2}}^{2}+\int_{t}^{t+1}\left(\|u(s)\|_{\mathbf{H}^{2}}^{2}+\|\phi(s)\|_{H^{3}}^{2}+\|\theta(s)\|_{H^{3}}^{2}\right) d s \leq \mathcal{D}(t),
$$

where $\mathcal{D}$ is a positive function depending on $\left\|u_{0}\right\|,\left\|\phi_{0}\right\|_{H^{1}},\left\|\theta_{0}\right\|_{H^{1}}, \Omega$, and coefficients of the system. In particular, $\lim _{t \rightarrow 0^{+}} \mathcal{D}(t)=+\infty$. 
3.2. Three dimensional case. First, we have the following lemma.

Lemma 3.7. Suppose $n=3$. Let $(u, \phi, \theta)$ be a smooth solution to problem (1.8)-(1.15). For the quantity $\mathcal{A}_{2}(t)$,

$$
\mathcal{A}_{2}(t)=\|\nabla u(t)\|^{2}+a \lambda_{0}\left\|\Delta \phi(t)-F^{\prime}(\phi(t))\right\|^{2}+\|\Delta \theta(t)\|^{2},
$$

the following differential inequality holds:

$$
\begin{aligned}
& \frac{d}{d t} \mathcal{A}_{2}(t)+\nu\|S u\|^{2}+a \lambda_{0} \gamma\left\|\nabla\left(\Delta \phi-F^{\prime}(\phi)\right)\right\|^{2}+k\|\nabla \Delta \theta\|^{2} \\
\leq & C_{*}\left(\mathcal{A}_{2}^{4}(t)+\mathcal{A}_{2}(t)\right), \quad \forall t>0 .
\end{aligned}
$$

$C_{*}$ is a positive constant which may depend on $\left\|u_{0}\right\|,\left\|\phi_{0}\right\|_{H^{1}},\left\|\theta_{0}\right\|_{H^{1}}, \Omega$, and coefficients of the system.

REMARK 3.1. We note that the coefficient of the third term in $\mathcal{A}_{2}(t)$ is different from the one in $\mathcal{A}_{1}(t)$ (see (3.1)).

Proof. We re-estimate the right-hand side of (3.3) and (3.5) by using the $3 D$ version of Sobolev embedding theorems. The estimates for $K_{3}$ and $K_{5}$ remain unchanged. Next,

$$
\begin{aligned}
& K_{1} \leq\|u\|_{\mathbf{L}^{\infty}}\|\nabla u\|\|S u\| \leq C\|S u\|^{\frac{3}{2}}\|\nabla u\|^{\frac{3}{2}} \leq \frac{\nu}{12}\|S u\|^{2}+C\|\nabla u\|^{6} . \\
& K_{2 a}+K_{2 b} \leq C\|\nabla \theta\|_{\mathbf{L}^{6}}\|\nabla \phi\|_{\mathbf{L}^{6}}^{2}\|S u\| \\
& \leq \frac{\nu}{12}\|S u\|^{2}+C\|\Delta \theta\|^{2}\|\Delta \phi\|^{4} \\
& \leq \frac{\nu}{12}\|S u\|^{2}+C\|\Delta \theta\|^{2}\left\|\Delta \phi-F^{\prime}(\phi)\right\|^{4}+C\|\Delta \theta\|^{2} . \\
& K_{2 c} \leq|b| \lambda_{0}\|\theta\|_{L^{\infty}}\|\Delta \phi\|\|\nabla \phi\|_{\mathbf{L}^{\infty}}\|S u\| \\
& \leq C\|\Delta \theta\|^{\frac{1}{2}}\|\nabla \theta\|^{\frac{1}{2}}\|\Delta \phi\|\|\nabla \phi\|_{\mathbf{H}^{2}}^{\frac{1}{2}}\|\Delta \phi\|^{\frac{1}{2}}\|S u\| \\
& \leq \frac{\nu}{12}\|S u\|^{2}+C\|\Delta \theta\|\|\nabla \theta\|\left(\left\|\Delta \phi-F^{\prime}(\phi)\right\|^{3}+1\right) \\
& \times\left(\left\|\nabla\left(\Delta \phi-F^{\prime}(\phi)\right)\right\|+\left\|\Delta \phi-F^{\prime}(\phi)\right\|+1\right) \\
& \leq \frac{\nu}{12}\|S u\|^{2}+\frac{a \lambda_{0} \gamma}{6}\left\|\nabla\left(\Delta \phi-F^{\prime}(\phi)\right)\right\|^{2} \\
& +C\left(\left\|\Delta \phi-F^{\prime}(\phi)\right\|^{2}+\left\|\Delta \phi-F^{\prime}(\phi)\right\|^{8}+\|\Delta \theta\|^{2}+\|\Delta \theta\|^{8}\right) . \\
& K_{4} \leq a \lambda_{0}\|\nabla \pi\|\|\nabla \phi\|_{\mathbf{L}^{6}}\left\|\Delta \phi-F^{\prime}(\phi)\right\|_{L^{3}} \\
& \leq C\|S u\|\left(\left\|\Delta \phi-F^{\prime}(\phi)\right\|+1\right)\left\|\Delta \phi-F^{\prime}(\phi)\right\|^{\frac{1}{2}}\left\|\nabla\left(\Delta \phi-F^{\prime}(\phi)\right)\right\|^{\frac{1}{2}} \\
& \leq \frac{\nu}{12}\|S u\|^{2}+\frac{a \lambda_{0} \gamma}{6}\left\|\nabla\left(\Delta \phi-F^{\prime}(\phi)\right)\right\|^{2} \\
& +C\left(\left\|\Delta \phi-F^{\prime}(\phi)\right\|^{2}+\left\|\Delta \phi-F^{\prime}(\phi)\right\|^{6}\right) . \\
& K_{6}=2 a \lambda_{0} \int_{\Omega} \nabla_{j}\left(\Delta \phi-F^{\prime}(\phi)\right) u_{i} \nabla_{j} \nabla_{i} \phi d x
\end{aligned}
$$




$$
\begin{aligned}
&-2 a \lambda_{0}\left(\nabla\left(\Delta \phi-F^{\prime}(\phi)\right), F^{\prime}(\phi) u\right) \\
& \leq C\left\|\nabla\left(\Delta \phi-F^{\prime}(\phi)\right)\right\|\left(\|u\|_{\mathbf{L}^{6}}\left\|\nabla^{2} \phi\right\|_{\mathbf{L}^{3}}+\left\|F^{\prime}(\phi)\right\|_{\mathbf{L}^{\infty}}\|u\|\right) \\
& \leq C\left\|\nabla\left(\Delta \phi-F^{\prime}(\phi)\right)\right\|\|\nabla u\| \\
& \times\left(\left\|\Delta \phi-F^{\prime}(\phi)\right\|^{\frac{1}{2}}\left\|\nabla\left(\Delta \phi-F^{\prime}\right)\right\|^{\frac{1}{2}}+\left\|\Delta \phi-F^{\prime}(\phi)\right\|+1\right) \\
& \leq \frac{a \lambda_{0} \gamma}{6}\left\|\nabla\left(\Delta \phi-F^{\prime}(\phi)\right)\right\|^{2} \\
& \quad+C\left(\|\nabla u\|^{2}+\|\nabla u\|^{8}+\left\|\Delta \phi-F^{\prime}(\phi)\right\|^{2}+\left\|\Delta \phi-F^{\prime}(\phi)\right\|^{8}\right) . \\
& K_{7} \leq \frac{k}{2}\|\nabla \Delta \theta\|^{2}+\frac{1}{k}\|\nabla(u \cdot \nabla \theta)\|^{2} \\
& \leq \frac{k}{2}\|\nabla \Delta \theta\|^{2}+C\left(\|\nabla u\|_{\mathbf{L}^{4}}^{2}\|\nabla \theta\|_{\mathbf{L}^{4}}^{2}+\|u\|_{\mathbf{L}^{\infty}}^{2}\|\Delta \theta\|^{2}\right) \\
& \leq \frac{k}{2}\|\nabla \Delta \theta\|^{2}+C\|S u\|^{\frac{3}{2}}\|\nabla u\|^{\frac{1}{2}}\|\Delta \theta\|^{2} \\
& \leq \frac{k}{2}\|\nabla \Delta \theta\|^{2}+\frac{\nu}{12}\|S u\|^{2}+C\|\nabla u\|^{2}\|\Delta \theta\|^{4} .
\end{aligned}
$$

Collecting all the estimates above, we arrive at our conclusion (3.9).

\section{Proof of Theorem 3.3.}

Proof. Due to Lemma 3.7, a standard argument of the ordinary differential equation yields that there is a time $T_{0}=T_{0}\left(u_{0}, \phi_{0}, \theta_{0}\right)<+\infty$ such that $\mathcal{A}_{2}(t)$ is bounded on $\left[0, T_{0}\right]$. This enables us to prove that problem (1.8)-(1.15) admits a local strong solution. Uniqueness of the strong solution follows from Proposition 3.9.

Since our problem contains the Navier-Stokes equation as a subsystem, in the $3 D$ case, we cannot expect the existence of global strong solutions to problem (1.8)-(1.15) for arbitrary large initial data. However, the global strong solution will exist if we further assume that the lower bound of the viscosity $\nu^{*}$ is sufficiently large.

Set

$$
\tilde{\mathcal{A}}_{2}(t)=\mathcal{A}_{2}(t)+1 .
$$

Then Theorem 3.4 is a direct consequence of the following higher-order differential inequality concerning $\tilde{\mathcal{A}}_{2}(t)$.

Lemma 3.8. Suppose $n=3$. We assume that $\nu \geq 1$ and (2.2) is fulfilled. Let $(u, \phi, \theta)$ be a smooth solution to the problem (1.8)-(1.15). Then the following inequality holds:

$$
\begin{aligned}
& \quad \frac{d}{d t} \tilde{\mathcal{A}}_{2}(t)+\left[\nu-M_{1} \nu^{\frac{1}{2}} \tilde{\mathcal{A}}_{2}(t)\right]\|S u\|^{2} \\
& \quad+\left(a \lambda_{0} \gamma-\frac{M_{1} \tilde{\mathcal{A}}_{2}(t)}{\nu^{\frac{1}{2}}}\right)\left\|\nabla\left(\Delta \phi-F^{\prime}(\phi)\right)\right\|^{2}+k\|\nabla \Delta \theta\|^{2} \\
& \leq M_{2} \mathcal{A}_{2}(t),
\end{aligned}
$$

where $M_{1}$ and $M_{2}$ are constants depending on $\left\|u_{0}\right\|,\left\|\phi_{0}\right\|_{H^{1}},\left\|\theta_{0}\right\|_{H^{1}}, \Omega$, and coefficients of the system, but not on $\nu$.

Proof. We note that the uniform estimates in Corollary 2.6 still hold. Then we re-estimate the terms $K_{1}, \ldots, K_{7}$ in an alternative way. The estimates for $K_{3}$ and $K_{5}$ 
remain unchanged. For the other terms, we have

$$
\begin{aligned}
K_{1} & \leq C\|S u\|^{\frac{7}{4}}\|u\|^{\frac{1}{4}}\|\nabla u\| \\
& \leq \frac{\nu}{12}\|S u\|^{2}+\nu^{\frac{1}{2}}\|\nabla u\|^{2}\|\Delta u\|^{2}+C \nu^{-\frac{11}{2}}\|\nabla u\|^{2},
\end{aligned}
$$

$$
\begin{aligned}
& \quad K_{2 a}+K_{2 b} \\
& \leq C\|\nabla \theta\|_{\mathbf{L}^{6}}\|\nabla \phi\|_{\mathbf{L}^{6}}^{2}\|S u\| \\
& \leq C\|\nabla \Delta \theta\|^{\frac{1}{2}}\|\nabla \theta\|^{\frac{1}{2}}\left(\left\|\Delta \phi-F^{\prime}(\phi)\right\|+1\right) \\
& \quad \times\left(\left\|\nabla\left(\Delta \phi-F^{\prime}(\phi)\right)\right\|^{\frac{1}{2}}\|\nabla \phi\|^{\frac{1}{2}}+1\right)\|S u\| \\
& \leq C\|\nabla \Delta \theta\|^{\frac{1}{2}}\|\nabla \theta\|^{\frac{1}{2}}\left(\left\|\Delta \phi-F^{\prime}(\phi)\right\|+1\right)\|S u\| \\
& \quad+C\|\nabla \Delta \theta\|^{\frac{1}{2}}\|\nabla \theta\|^{\frac{1}{2}}\left(\left\|\Delta \phi-F^{\prime}(\phi)\right\|+1\right)\|S u\|\left\|\nabla\left(\Delta \phi-F^{\prime}(\phi)\right)\right\|^{\frac{1}{2}} \\
& \leq\left[\frac{\nu}{12}+\nu^{\frac{1}{2}}\left(\left\|\Delta \phi-F^{\prime}(\phi)\right\|+\|\nabla \theta\|\right)\right]\|S u\|^{2}+\frac{k}{4}\|\nabla \Delta \theta\|^{2} \\
& \quad+\frac{C}{\nu}\left(1+\left\|\Delta \phi-F^{\prime}(\phi)\right\|^{2}\right)\left\|\nabla\left(\Delta \phi-F^{\prime}(\phi)\right)\right\|^{2} \\
& \quad+C\left(\frac{1}{\nu}+\frac{1}{\nu^{2}}\right)\left(\|\nabla \theta\|^{2}+\left\|\Delta \phi-F^{\prime}(\phi)\right\|^{2}\right),
\end{aligned}
$$

$$
\begin{aligned}
& K_{2 c} \leq|b| \lambda_{0}\|\theta\|_{L^{\infty}}\|\Delta \phi\|\|\nabla \phi\|_{\mathbf{L}^{\infty}}\|S u\| \\
& \leq C\|\Delta \theta\|^{\frac{1}{2}}\|\nabla \theta\|^{\frac{1}{2}}\|\Delta \phi\|^{\frac{3}{2}}\left(\|\nabla \Delta \phi\|^{\frac{1}{2}}+\|\Delta \phi\|^{\frac{1}{2}}\right)\|S u\| \\
& \leq C\|\Delta \theta\|^{\frac{1}{2}}\|\nabla \theta\|^{\frac{1}{2}}\left(\left\|\Delta \phi-F^{\prime}(\phi)\right\|^{\frac{3}{2}}+1\right)\left(\left\|\nabla\left(\Delta \phi-F^{\prime}(\phi)\right)\right\|^{\frac{1}{2}}+1\right)\|S u\| \\
& \leq\left[\frac{\nu}{12}+\nu^{\frac{1}{2}}\left(\left\|\Delta \phi-F^{\prime}(\phi)\right\|^{2}+\|\Delta \theta\|\right)\right]\|S u\|^{2} \\
& \quad+\left(\frac{a \lambda_{0} \gamma}{6}+\frac{1}{\nu}\left\|\Delta \phi-F^{\prime}(\phi)\right\|^{2}\right)\left\|\nabla\left(\Delta \phi-F^{\prime}(\phi)\right)\right\|^{2} \\
& \quad+C\left(1+\frac{1}{\nu}\right)\left(\|\Delta \theta\|^{2}+\left\|\Delta \phi-F^{\prime}(\phi)\right\|^{2}\right),
\end{aligned}
$$

$$
\begin{gathered}
K_{4} \leq C\|S u\|\left(\left\|\Delta \phi-F^{\prime}(\phi)\right\|+1\right)\left\|\Delta \phi-F^{\prime}(\phi)\right\|^{\frac{1}{2}}\left\|\nabla\left(\Delta \phi-F^{\prime}(\phi)\right)\right\|^{\frac{1}{2}} \\
\leq\left(\frac{\nu}{12}+\nu^{\frac{1}{2}}\left\|\Delta \phi-F^{\prime}(\phi)\right\|^{2}\right)\|S u\|^{2} \\
\quad+\frac{a \lambda_{0} \gamma}{6}\left\|\nabla\left(\Delta \phi-F^{\prime}(\phi)\right)\right\|^{2}+\frac{C}{\nu}\left\|\Delta \phi-F^{\prime}(\phi)\right\|^{2}, \\
K_{6} \leq C\left\|\Delta \phi-F^{\prime}(\phi)\right\|_{L^{3}}\|\nabla u\|_{L^{6}}\|\Delta \phi\| \\
\leq C\left\|\nabla\left(\Delta \phi-F^{\prime}(\phi)\right)\right\|^{\frac{1}{2}}\left\|\Delta \phi-F^{\prime}(\phi)\right\|^{\frac{1}{2}}\|S u\|\left(\left\|\Delta \phi-F^{\prime}(\phi)\right\|+1\right) \\
\leq\left(\frac{\nu}{12}+\nu^{\frac{1}{2}}\left\|\Delta \phi-F^{\prime}(\phi)\right\|\right)\|S u\|^{2} \\
\quad+\left(\frac{a \lambda_{0} \gamma}{6}+\frac{1}{\nu}\left\|\Delta \phi-F^{\prime}(\phi)\right\|^{2}\right)\left\|\nabla\left(\Delta \phi-F^{\prime}(\phi)\right)\right\|^{2} \\
\quad+C\left(1+\frac{1}{\nu^{2}}\right)\left\|\Delta \phi-F^{\prime}(\phi)\right\|^{2},
\end{gathered}
$$

$K_{7} \leq \frac{k}{2}\|\nabla \Delta \theta\|^{2}+C\left(\|\nabla u\|_{\mathbf{L}^{4}}^{2}\|\nabla \theta\|_{\mathbf{L}^{4}}^{2}+\|u\|_{\mathbf{L}^{\infty}}^{2}\|\Delta \theta\|^{2}\right)$ 


$$
\begin{aligned}
& \leq \frac{k}{2}\|\nabla \Delta \theta\|^{2}+C\|S u\|^{\frac{3}{2}}\|\nabla u\|^{\frac{1}{2}}\|\Delta \theta\|^{\frac{3}{2}}\|\nabla \theta\|^{\frac{1}{2}}+C\|S u\|^{\frac{3}{2}}\|u\|^{\frac{1}{2}}\|\Delta \theta\|^{2} \\
& \leq \frac{k}{2}\|\nabla \Delta \theta\|^{2}+\nu^{\frac{1}{2}}\|\Delta \theta\|^{2}\|S u\|^{2}+\frac{C}{\nu^{\frac{3}{2}}}\left(\|\nabla u\|^{2}+\|\Delta \theta\|^{2}\right) .
\end{aligned}
$$

Combining the above estimates, using the fact $\nu \geq 1$ and the definition of $\tilde{\mathcal{A}}_{2}(t)$, we deduce the inequality (3.10). The proof is complete.

\section{Proof of Theorem 3.4.}

Proof. It follows from Corollary 2.6 that

$$
\int_{t}^{t+1} \mathcal{A}_{2}(\tau) d \tau \leq \tilde{M}, \quad \forall t \geq 0
$$

where $\tilde{M}>0$ may depend on $\Omega,\left\|u_{0}\right\|,\left\|\phi_{0}\right\|_{H^{1}},\left\|\theta_{0}\right\|_{H^{1}}$, and coefficients of the system except $\nu$. Moreover, if the viscosity $\nu$ is sufficiently large such that

$$
\nu^{\frac{1}{2}} \geq \max \left\{1, \frac{1}{a \lambda_{0} \gamma}\right\} M_{1}\left(\tilde{\mathcal{A}}_{2}(0)+M_{2} \tilde{M}+2 \tilde{M}\right)+1,
$$

then following the same argument as in $[17,31]$, we can use Lemma 3.8 to obtain the uniform estimate

$$
\tilde{\mathcal{A}}_{2}(t) \leq \frac{\nu^{\frac{1}{2}} \min \left\{a \lambda_{0} \gamma, 1\right\}}{M_{1}}, \quad \forall t \geq 0,
$$

which yields the required conclusion.

3.3. Uniqueness of strong solutions. The uniqueness of strong solutions to the problem (1.8)-(1.15) can be obtained by the energy method.

Proposition 3.9 (Uniqueness of strong solutions). For $n=2,3$, let $\left(u_{1}, \phi_{1}, \theta_{1}\right)$ and $\left(u_{2}, \phi_{2}, \theta_{2}\right)$ be two strong solutions on $[0, T]$ that start from the same initial data $\left(u_{0}, \phi_{0}, \theta_{0}\right) \in \mathbf{V} \times H^{2}(\Omega) \times\left(H_{0}^{1}(\Omega) \cap H^{2}(\Omega)\right)$ satisfying (2.2). Then $\left(u_{1}, \phi_{1}, \theta_{1}\right)=$ $\left(u_{2}, \phi_{2}, \theta_{2}\right)$.

Proof. We provide the proof for $3 D$ case and the proof for $2 D$ is similar. Denote

$$
\bar{u}=u_{1}-u_{2}, \quad \bar{\phi}=\phi_{1}-\phi_{2}, \quad \bar{\theta}=\theta_{1}-\theta_{2} .
$$

We can see that $(\bar{u}, \bar{\phi}, \bar{\theta})$ satisfy

$$
\begin{aligned}
&\left\langle\bar{u}_{t}, v\right\rangle_{\mathbf{V}^{\prime}, \mathbf{V}}+\int_{\Omega}\left[\left(u_{1} \cdot \nabla\right) u_{1}-\left(u_{2} \cdot \nabla\right) u_{2}\right] \cdot v d x+\nu \int_{\Omega} \nabla \bar{u}: \nabla \bar{v} d x \\
&= \int_{\Omega}\left[\lambda\left(\theta_{1}\right) \nabla \phi_{1} \otimes \nabla \phi_{1}-\lambda\left(\theta_{2}\right) \nabla \phi_{2} \otimes \nabla \phi_{2}\right]: \nabla v d x \\
&+\alpha g \int_{\Omega} \bar{\theta} \mathbf{j} \cdot v d x, \quad \forall v \in \mathbf{V}, \\
& \bar{\phi}_{t}+u_{1} \cdot \nabla \phi_{1}-u_{2} \cdot \nabla \phi_{2}=\gamma\left(\Delta \bar{\phi}-F^{\prime}\left(\phi_{1}\right)+F^{\prime}\left(\phi_{2}\right)\right), \quad \text { a.e. in } \Omega, \\
& \bar{\theta}_{t}+u_{1} \cdot \nabla \theta_{1}-u_{2} \cdot \nabla \theta_{2}=k \Delta \bar{\theta}, \quad \text { a.e. in } \Omega .
\end{aligned}
$$


Taking $v=\bar{u}$ in (3.14), testing (3.15) by $-a \lambda_{0} \Delta \bar{\phi}$ and (3.16) by $-\Delta \bar{\theta}$ in $L^{2}(\Omega)$, respectively, adding up these three resultants, then performing integration by parts and using the incompressible condition for the velocity, we get

$$
\begin{aligned}
& \begin{array}{l}
\frac{1}{2} \frac{d}{d t}\left(\|\bar{u}\|^{2}+a \lambda_{0}\|\nabla \bar{\phi}\|^{2}+\|\nabla \bar{\theta}\|^{2}\right)+\nu\|\nabla \bar{u}\|^{2}+a \lambda_{0} \gamma\|\Delta \bar{\phi}\|^{2}+k\|\Delta \bar{\theta}\|^{2} \\
=-\left(\bar{u} \cdot \nabla u_{1}, \bar{u}\right)-a \lambda_{0}\left(\nabla \bar{\phi} \Delta \phi_{1}, \bar{u}\right)+a \lambda_{0}\left(u_{1} \cdot \nabla \bar{\phi}, \Delta \bar{\phi}\right)-b \lambda_{0}\left(\bar{\theta} \nabla \phi_{1} \otimes \nabla \phi_{1}, \nabla \bar{u}\right) \\
\quad-b \lambda_{0}\left(\theta_{2} \nabla \bar{\phi} \otimes \nabla \phi_{1}, \nabla \bar{u}\right)-b \lambda_{0}\left(\theta_{2} \nabla \phi_{2} \otimes \nabla \bar{\phi}, \nabla \bar{u}\right)+\alpha g(\bar{\theta} \mathbf{j}, \bar{u}) \\
\quad+a \lambda_{0} \gamma\left(F^{\prime}\left(\phi_{1}\right)-F^{\prime}\left(\phi_{2}\right), \Delta \bar{\phi}\right)+\left(\bar{u} \cdot \nabla \theta_{1}, \Delta \bar{\theta}\right)+\left(u_{2} \cdot \nabla \bar{\theta}, \Delta \bar{\theta}\right)
\end{array} \\
&=\sum_{m=1}^{10} I_{m} .
\end{aligned}
$$

Keeping in mind the uniform estimates obtained in Corollary 2.6, we proceed to estimate the right hand side of (3.17),

$$
\begin{aligned}
& I_{1} \leq\|\bar{u}\|_{\mathbf{L}^{4}}^{2}\left\|\nabla u_{1}\right\| \leq C\|\nabla \bar{u}\|^{\frac{3}{2}}\|\bar{u}\|^{\frac{1}{2}}\left\|\nabla u_{1}\right\| \\
& \leq \frac{\nu}{12}\|\nabla \bar{u}\|^{2}+C\left\|\nabla u_{1}\right\|^{4}\|\bar{u}\|^{2} . \\
& I_{2} \leq a \lambda_{0}\|\nabla \bar{\phi}\|_{\mathbf{L}^{6}}\|\bar{u}\|_{\mathbf{L}^{3}}\left\|\Delta \phi_{1}\right\| \leq C\left\|\Delta \phi_{1}\right\|\|\bar{u}\|^{\frac{1}{2}}\|\nabla \bar{u}\|^{\frac{1}{2}}\|\Delta \bar{\phi}\| \\
& \leq \frac{\nu}{12}\|\nabla \bar{u}\|^{2}+\frac{a \lambda_{0} \gamma}{10}\|\Delta \bar{\phi}\|^{2}+C\left\|\Delta \phi_{1}\right\|^{4}\|\bar{u}\|^{2} . \\
& I_{3} \leq\left\|u_{1}\right\|_{\mathbf{L}^{6}}\|\Delta \bar{\phi}\|\|\nabla \bar{\phi}\|_{\mathbf{L}^{3}} \leq C\left\|\nabla u_{1}\right\|\|\Delta \bar{\phi}\|^{\frac{3}{2}}\|\nabla \bar{\phi}\|^{\frac{1}{2}} \\
& \leq \frac{a \lambda_{0} \gamma}{10}\|\Delta \bar{\phi}\|^{2}+C\left\|\nabla u_{1}\right\|^{4}\|\nabla \bar{\phi}\|^{2} \text {. } \\
& I_{4}+I_{7} \leq|b| \lambda_{0}\|\nabla \bar{u}\|\|\bar{\theta}\|_{L^{\infty}}\left\|\nabla \phi_{1}\right\|_{\mathbf{L}^{4}}^{2}+|\alpha\|g \mid\| \bar{\theta}\|\| \bar{u} \| \\
& \leq C\|\nabla \bar{u}\|\|\nabla \bar{\theta}\|^{\frac{1}{2}}\|\Delta \bar{\theta}\|^{\frac{1}{2}}+C\|\nabla \bar{u}\|\|\nabla \bar{\theta}\| \\
& \leq \frac{\nu}{12}\|\nabla \bar{u}\|^{2}+\frac{k}{6}\|\Delta \bar{\theta}\|^{2}+C\|\nabla \bar{\theta}\|^{2} . \\
& I_{5} \leq|b| \lambda_{0}\left\|\theta_{2}\right\|_{L^{\infty}}\|\nabla \bar{u}\|\|\nabla \bar{\phi}\|_{\mathbf{L}^{3}}\left\|\nabla \phi_{1}\right\|_{\mathbf{L}^{6}} \\
& \leq C\|\nabla \bar{u}\|\|\Delta \bar{\phi}\|^{\frac{1}{2}}\|\nabla \bar{\phi}\|^{\frac{1}{2}}\left\|\Delta \phi_{1}\right\| \\
& \leq \frac{\nu}{12}\|\nabla \bar{u}\|^{2}+\frac{a \lambda_{0} \gamma}{10}\|\Delta \bar{\phi}\|^{2}+C\left\|\Delta \phi_{1}\right\|^{4}\|\nabla \bar{\phi}\|^{2} . \\
& I_{6} \leq|b| \lambda_{0}\left\|\theta_{2}\right\|_{L^{\infty}}\|\nabla \bar{u}\|\|\nabla \bar{\phi}\|_{\mathbf{L}^{3}}\left\|\nabla \phi_{2}\right\|_{\mathbf{L}^{6}} \\
& \leq C\|\nabla \bar{u}\|\|\Delta \bar{\phi}\|^{\frac{1}{2}}\|\nabla \bar{\phi}\|^{\frac{1}{2}}\|\| \Delta \phi_{2} \| \\
& \leq \frac{\nu}{12}\|\nabla \bar{u}\|^{2}+\frac{a \lambda_{0} \gamma}{10}\|\Delta \bar{\phi}\|^{2}+C\left\|\Delta \phi_{2}\right\|^{4}\|\nabla \bar{\phi}\|^{2} . \\
& I_{8} \leq a \lambda_{0} \gamma\left\|\bar{\phi} \int_{0}^{1} F^{\prime \prime}\left(s \phi_{1}+(1-s) \phi_{2}\right) d s\right\|_{L^{\infty}}\|\Delta \bar{\phi}\|
\end{aligned}
$$




$$
\begin{aligned}
& \leq C\left\|F^{\prime \prime}\right\|_{L^{\infty}}\|\bar{\phi}\|_{L^{\infty}}\|\Delta \bar{\phi}\| \\
& \leq C\|\Delta \bar{\phi}\|^{\frac{3}{2}}\|\nabla \bar{\phi}\|^{\frac{1}{2}} \\
& \leq \frac{a \lambda_{0} \gamma}{10}\|\Delta \bar{\phi}\|^{2}+C\|\nabla \bar{\phi}\|^{2} . \\
& I_{9} \leq\|\bar{u}\|_{\mathbf{L}^{3}}\left\|\nabla \theta_{1}\right\|_{\mathbf{L}^{6}}\|\Delta \bar{\theta}\| \leq C\left\|\Delta \theta_{1}\right\|\|\bar{u}\|^{\frac{1}{2}}\|\nabla \bar{u}\|^{\frac{1}{2}}\|\Delta \bar{\theta}\| \\
& \leq \frac{\nu}{12}\|\nabla \bar{u}\|^{2}+\frac{k}{6}\|\Delta \bar{\theta}\|^{2}+C\left\|\Delta \theta_{1}\right\|^{4}\|\bar{u}\|^{2} . \\
& I_{10} \leq\left\|u_{2}\right\|_{\mathbf{L}^{6}}\|\nabla \bar{\theta}\|_{\mathbf{L}^{3}}\|\Delta \bar{\theta}\| \leq C\left\|\nabla u_{2}\right\|\|\nabla \bar{\theta}\|^{\frac{1}{2}}\|\Delta \bar{\theta}\|^{\frac{3}{2}} \\
& \leq \frac{k}{6}\|\Delta \bar{\theta}\|^{2}+C\left\|\nabla u_{2}\right\|^{4}\|\nabla \bar{\theta}\|^{2} .
\end{aligned}
$$

Summing up, we obtain that

$$
\begin{aligned}
& \frac{d}{d t}\left(\|\bar{u}\|^{2}+a \lambda_{0}\|\nabla \bar{\phi}\|^{2}+\|\nabla \bar{\theta}\|^{2}\right)+\nu\|\nabla \bar{u}\|^{2}+a \lambda_{0} \gamma\|\Delta \bar{\phi}\|^{2}+k\|\Delta \bar{\theta}\|^{2} \\
\leq & Q(t)\left(\|\bar{u}\|^{2}+a \lambda_{0}\|\nabla \bar{\phi}\|^{2}+\|\nabla \bar{\theta}\|^{2}\right),
\end{aligned}
$$

where

$$
Q(t)=C\left(1+\left\|\nabla u_{1}\right\|^{4}+\left\|\Delta \phi_{1}\right\|^{4}+\left\|\Delta \theta_{1}\right\|^{4}+\left\|\nabla u_{2}\right\|^{4}+\left\|\Delta \phi_{2}\right\|^{4}\right)
$$

with $C$ being a constant that may depend on $M, \Omega$, and coefficients of the system. Since the $\mathbf{V} \times H^{2} \times H^{2}$-norm of strong solutions to problem (1.8)-(1.15) are bounded on its existence time interval, $Q(t)$ is bounded on $[0, T]$. Then we can complete the proof by applying the Gronwall inequality.

\section{REMARK 3.2 .}

(1) If $n=2$, it is easy to check that (3.18) holds with

$$
Q(t)=C\left(1+\left\|\nabla u_{1}\right\|^{2}+\left\|\Delta \phi_{1}\right\|^{2}+\left\|\Delta \theta_{1}\right\|^{2}+\left\|\nabla u_{2}\right\|^{2}+\left\|\Delta \phi_{2}\right\|^{2}\right) .
$$

On the other hand, Corollary 2.6 implies that

$$
\int_{t}^{t+1} Q(s) d s \leq C, \quad \forall t \geq 0 .
$$

As a consequence, we can actually obtain the uniqueness of weak solutions to the problem (1.8)-(1.15) for the two dimensional case.

(2) It is interesting to ask whether the problem (1.8)-(1.15) has a weak-strong uniqueness result in $3 D$ as for the Navier-Stokes equations (c.f. Serrin [24]). The main difficulty here is that due to the temperature-dependence of the surface tension coefficient $\lambda$, we lose some dissipation in the derivation of the basic energy inequality (2.3) in order to control the corresponding higher-order nonlinear stress terms. When $\lambda$ is a constant, one can obtain the weak-strong uniqueness result as in [17] for a simplified liquid crystal system. 


\section{Long-time dynamics and stability}

In this section, we shall discuss the long-time behavior of global weak/strong solutions and stability properties of the problem (1.8)-(1.15). First, we present an alternative result that indicates the eventual regularity of weak solutions in $3 D$ and is helpful to understand the long-time behavior of global solutions.

Proposition 4.1. Suppose $n=3$. For any $\left(u_{0}, \phi_{0}, \theta_{0}\right) \in \mathbf{V} \times H^{2}(\Omega) \times\left(H_{0}^{1}(\Omega) \cap\right.$ $\left.H^{2}(\Omega)\right)$ satisfying $(2.2)$ and

$$
\mathcal{A}_{2}(0)=\left\|\nabla u_{0}\right\|^{2}+a \lambda_{0}\left\|\Delta \phi_{0}-F^{\prime}\left(\phi_{0}\right)\right\|^{2}+\left\|\Delta \theta_{0}\right\|^{2} \leq R,
$$

where $R>0$ is a constant, there exists $\varepsilon_{0}>0$ depending on $\left\|u_{0}\right\|,\left\|\phi_{0}\right\|_{H^{1}},\left\|\theta_{0}\right\|_{H^{1}}, \Omega$, $R$, and coefficients of the system such that either

(i) the problem (1.8)-(1.15) admits a unique global strong solution that is uniformly bounded in time in $\mathbf{V} \times H^{2} \times\left(H_{0}^{1} \cap H^{2}\right)$, or

(ii) there is a $T_{*} \in(0,+\infty)$ such that $\mathcal{E}\left(T_{*}\right)<\mathcal{E}(0)-\varepsilon_{0}$.

Proof. The proof follows from the idea in [17]. For the convenience of the readers, we sketch it here. Recalling the higher-order differential inequality (3.9), we consider the following initial value problem of an ordinary differential equation:

$$
\frac{d}{d t} Y(t)=C_{*}\left(Y^{4}(t)+Y(t)\right), \quad Y(0)=R \geq \mathcal{A}_{2}(0) .
$$

We denote by $I=\left[0, T_{\max }\right)$ the maximal existence interval of $Y(t)$ such that $\lim _{t \rightarrow T_{\text {max }}^{-}} Y(t)=+\infty$. It is easy to check that

$$
0 \leq \mathcal{A}_{2}(t) \leq Y(t), \quad \forall t \in I
$$

which indicates that $\mathcal{A}(t)$ exists on $I$. We note that $T_{\text {max }}$ is determined by $Y(0)=R$ and $C_{*}$ such that $T_{\max }=T_{\max }\left(R, C_{*}\right)$ is increasing when $R$ is decreasing. Taking $t_{0}=\frac{3}{4} T_{\max }\left(R, C_{*}\right)>0$, then we have

$$
0 \leq \mathcal{A}_{2}(t) \leq Y(t) \leq K, \quad \forall t \in\left[0, t_{0}\right]
$$

where $K$ is a constant that only depends on $R, C_{*}, t_{0}$. Take

$$
\varepsilon_{0}=\frac{1}{3} R t_{0} \min \{\nu, \gamma, k\}
$$

If (ii) is not true, namely, $\mathcal{E}(t) \geq \mathcal{E}(0)-\varepsilon_{0}$ for all $t \geq 0$, we infer from (2.3) that

$$
\int_{0}^{+\infty}\left(\frac{\nu}{2}\|\nabla u\|^{2}+a \lambda_{0} \gamma\left\|\Delta \phi-F^{\prime}(\phi)\right\|^{2}+k\|\Delta \theta\|^{2}\right) d t \leq \varepsilon_{0}
$$

Hence, there exists a $t_{*} \in\left[\frac{2}{3} t_{0}, t_{0}\right]$ such that

$$
\mathcal{A}_{2}\left(t_{*}\right) \leq \max \left\{\frac{2}{\nu}, \frac{1}{\gamma}, \frac{1}{k}\right\} \frac{3 \varepsilon_{0}}{t_{0}}=R .
$$

Taking $t_{*}$ as the initial time and $Y\left(t_{*}\right)=R$ in (4.2), then it follows from the above argument that $Y(t)$ (and thus $\mathcal{A}_{2}(t)$ ) is uniformly bounded at least on $\left[0, t_{*}+t_{0}\right] \supset$ 
$\left[0, \frac{5}{3} t_{0}\right]$. Its bound remains the same as that on $\left[0, t_{0}\right]$. By iteration, it follows that $\mathcal{A}_{2}(t)$ is uniformly bounded for $t \geq 0$. Thus, we can extend the (unique) local strong solution to infinity to get a global one.

Proposition 4.2 (Eventual regularity of weak solutions in $3 D$ ). When $n=3$, let $(u, \phi, \theta)$ be a global weak solution of the problem (1.8)-(1.15). Then there exists a time $T_{0} \in(0,+\infty)$ such that $(u, \phi, \theta)$ becomes a strong solution in $\left[T_{0},+\infty\right)$.

Proof. We simply take

$$
R=1, \quad t_{0}=\frac{3}{4} T_{\max }\left(R, C_{*}\right), \quad \varepsilon_{0}=\frac{1}{3} t_{0} \min \left\{\frac{\nu}{2}, \gamma, k\right\}
$$

in the proof of Proposition 4.1. It follows from (2.3) that there exist a $T_{1}>0$ such that

$$
\int_{T_{1}}^{+\infty}\left(\frac{\nu}{2}\|\nabla u\|^{2}+a \lambda_{0} \gamma\left\|\Delta \phi-F^{\prime}(\phi)\right\|^{2}+k\|\Delta \theta\|^{2}\right) d t \leq \varepsilon_{0}
$$

Then we can find a time $T_{0} \in\left[T_{1}, T_{1}+\frac{1}{3} t_{0}\right]$ such that $\mathcal{A}_{2}\left(T_{0}\right) \leq 1$ and

$$
\mathcal{E}(t)-\mathcal{E}\left(T_{0}\right) \geq \mathcal{E}(t)-\mathcal{E}\left(T_{1}\right) \geq-\varepsilon_{0}, \quad \forall t \geq T_{0} .
$$

Taking $T_{0}$ as the initial time, we can apply Proposition 4.1. The proof is complete.

4.1. Convergence to equilibrium. We shall show the convergence of global solutions to single steady states as time tends to infinity. Let $(u, \phi, \theta)$ be a global weak solution of the problem (1.8)-(1.15). We infer from either Proposition $3.6(n=2)$ or Proposition $4.2(n=3)$ that after a certain time $T>0$, the weak solution will be a strong one that is uniformly bounded in $\mathbf{V} \times H^{2} \times H^{2}$ for all $t \geq T$. Since we are now considering the long-time behavior as $t \rightarrow+\infty$, we can simply use a shift in time and reduce our study to the case of bounded strong solutions.

The main result of this subsection is as follows.

Theorem 4.3. Suppose $n=2,3$. Any bounded global strong solution $(u, \phi, \theta)$ of the problem (1.8)-(1.15) converges to a steady state $\left(\mathbf{0}, \phi_{\infty}, 0\right)$ as time goes to infinity such that

$$
\lim _{t \rightarrow+\infty}\left(\|u(t)\|_{\mathbf{H}^{1}}+\left\|\phi(t)-\phi_{\infty}\right\|_{H^{2}}+\|\theta(t)\|_{H^{2}}\right)=0,
$$

where $\phi_{\infty}$ satisfies the following nonlinear elliptic boundary value problem:

$$
-\Delta \phi_{\infty}+F^{\prime}\left(\phi_{\infty}\right)=0, \quad x \in \Omega, \text { with }\left.\phi_{\infty}\right|_{\Gamma}=-1 .
$$

Moreover, we have the convergence rate

$$
\|u(t)\|_{\mathbf{H}^{1}}+\left\|\phi(t)-\phi_{\infty}\right\|_{H^{2}}+\|\theta(t)\|_{H^{2}} \leq C(1+t)^{-\frac{\xi}{(1-2 \xi)}}, \quad \forall t \geq 0 .
$$

$\xi \in\left(0, \frac{1}{2}\right)$ is a constant depending on $\phi_{\infty}$. Furthermore, $\theta$ satisfies an exponential decay such that there exists a constant $C_{0}=C_{0}(n, \Omega)>0$,

$$
\|\theta(t)\| \leq\left\|\theta_{0}\right\| e^{-C_{0} t}, \quad \forall t \geq 0 .
$$


Remark 4.1. Decay properties of the velocity $u$ and temperature $\theta$ as time tends to infinity can be obtained by the energy method (see Proposition 4.4 below). However, convergence for the phase function $\phi$ is usually nontrivial because the structure of the set of equilibria may be complicated and the solutions to elliptic problem like (4.6) may form a continuum if the spatial dimension $n \geq 2$ (cf. e.g., Haraux [12, Remark 2.3.13]). As we have mentioned in the introduction, our results and their proofs hold for general Dirichlet boundary data for the phase function such that -1 in (1.14) can be replaced by a certain generic function $h(x)$. Since our problem enjoys a dissipative energy inequality (2.3), we can achieve the goal by using the Łojasiewicz-Simon approach (cf. e.g., $[9,13,14,25]$ ). One advantage of this approach is that we can obtain the convergence result without investigating the structure of equilibria.

The $\omega$-limit set of $\left(u_{0}, \phi_{0}, \theta_{0}\right) \in \mathbf{V} \times H^{2}(\Omega) \times\left(H_{0}^{1}(\Omega) \cap H^{2}(\Omega)\right)$ is defined as follows:

$$
\begin{aligned}
& \omega\left(u_{0}, \phi_{0}, \theta_{0}\right)=\left\{\left(u_{\infty}(x), \phi_{\infty}(x), \theta_{\infty}(x)\right) \in \mathbf{V} \times H^{2} \times\left(H^{2} \cap H_{0}^{1}\right):\right. \\
& \quad \text { there exists }\left\{t_{n}\right\} \nearrow \varnothing \text { such that } \\
& \left.\left(u\left(t_{n}\right), \phi\left(t_{n}\right), \theta\left(t_{n}\right)\right) \rightarrow\left(u_{\infty}, \phi_{\infty}, \theta_{\infty}\right) \text { in } \mathbf{L}^{2} \times H^{1} \times H^{1}\right\} .
\end{aligned}
$$

Proposition 4.4. Let $n=2,3$. For any global strong solutions to problem (1.8)(1.15), there exists $\mathcal{E}_{\infty} \geq 0$ such that

$$
\lim _{t \rightarrow+\infty} \mathcal{E}(t)=\mathcal{E}_{\infty}
$$

and it holds that

$$
\lim _{t \rightarrow+\infty}\left(\|u(t)\|_{\mathbf{H}^{1}}+\left\|\Delta \phi(t)-F^{\prime}(\phi(t))\right\|+\|\Delta \theta(t)\|\right)=0 .
$$

Proof. The total energy $\mathcal{E}(t)$ is nonnegative and decreasing as $t$ increases (cf. (2.3)). Then (4.9) easily follows. For a global bounded strong solution $(u, \phi, \theta)$, we have $\mathcal{A}_{1}(t) \leq C(n=2)$ or $\mathcal{A}_{2}(t) \leq C(n=3)$, so it follows from Lemma $3.5(n=2)$ or Lemma $3.7(n=3)$ that $\frac{d \mathcal{A}_{i}(t)}{d t} \leq C(i=1,2)$. On the other hand, we know from (2.3) that $\mathcal{A}_{i}(t) \in L^{1}(0,+\infty)$. As a result, we can infer from Zheng [36, Lemma 6.2.1] that $\lim _{t \rightarrow+\infty} \mathcal{A}_{i}(t)=0$, which yields (4.10).

COROLlary 4.5. $\omega\left(u_{0}, \phi_{0}, \theta_{0}\right)$ is a nonempty bounded subset in $\mathbf{V} \times H^{2} \times\left(H^{2} \cap H_{0}^{1}\right)$. Moreover, $\omega\left(u_{0}, \phi_{0}, \theta_{0}\right) \subset \mathcal{S}=\left\{(\mathbf{0}, \tilde{\phi}, 0) \mid-\Delta \tilde{\phi}+F^{\prime}(\tilde{\phi})=0\right.$ in $\left.\Omega,\left.\tilde{\phi}\right|_{\Gamma}=-1\right\}$ and $\mathcal{E}=\mathcal{E}_{\infty}$ on $\omega\left(u_{0}, \phi_{0}, \theta_{0}\right)$.

It is easy to verify that a critical point of the elastic energy $E(\phi)$ given by (1.7) is equivalent to a solution to the following elliptic boundary value problem:

$$
-\Delta \phi+F^{\prime}(\phi)=0, \quad x \in \Omega,\left.\quad \phi\right|_{\Gamma}=-1 .
$$

We recall the following Łojasiewicz-Simon type inequality (cf. [13]).

Lemma 4.6 (Lojasiewicz-Simon inequality). Let $\psi$ be a critical point of $E(\phi)$. Then there exist constants $\xi \in\left(0, \frac{1}{2}\right)$ and $\beta>0$ depending on $\psi$ such that for any $\phi \in$ $H^{1}(\Omega),\left.\phi\right|_{\Gamma}=-1$ satisfying $\|\phi-\psi\|_{H^{1}(\Omega)}<\beta$, it holds that

$$
\left\|-\Delta \phi+F^{\prime}(\phi)\right\|_{H^{-1}} \geq|E(\phi)-E(\psi)|^{1-\xi} .
$$


For any global bounded strong solution, it follows from Corollary 4.5 that there is an increasing unbounded sequence $\left\{t_{n}\right\}_{n \in \mathbb{N}}$ and a function $\phi_{\infty} \in \mathcal{S}$ such that

$$
\lim _{t_{n} \rightarrow+\infty}\left\|\phi\left(t_{n}\right)-\phi_{\infty}\right\|_{H^{1}}=0
$$

As a result, we infer from Proposition 4.4 and (4.13) that

$$
\lim _{t_{n} \rightarrow+\infty} \mathcal{E}\left(t_{n}\right)=a \lambda_{0} E\left(\phi_{\infty}\right)=\mathcal{E}_{\infty} \text { and } \mathcal{E}(t) \geq a \lambda_{0} E\left(\phi_{\infty}\right), \forall t>0 .
$$

If $\mathcal{E}\left(t_{*}\right)=a \lambda_{0} E\left(\phi_{\infty}\right)$ for some $t_{*}>0$, then $\mathcal{E}(t)=\mathcal{E}_{\infty}$ for all $t \geq 0$. Thus, by (2.3), we have $\|u(t)\|_{\mathbf{v}}=\|\theta(t)\|_{H^{2}}=\left\|\Delta \phi(t)-F^{\prime}(\phi(t))\right\|=0$ for $t \geq t_{0}$. Besides, it follows from equation (1.10) that

$$
\left\|\phi_{t}\right\| \leq\|u\|_{\mathbf{L}^{4}}\|\nabla \phi\|_{\mathbf{L}^{4}}+\left\|\Delta \phi-F^{\prime}(\phi)\right\| \leq C\left(\|\nabla u\|+\left\|\Delta \phi-F^{\prime}(\phi)\right\|\right),
$$

thus $\left\|\phi_{t}(t)\right\|=0$ for $t \geq t_{*}$, and due to (4.13), we have $\phi(t)=\phi_{\infty}$ for $t \geq t_{0}$.

Then we only have to consider the case $\mathcal{E}(t)>\mathcal{E}_{\infty}=a \lambda_{0} E\left(\phi_{\infty}\right)$, for all $t>0$. Based on the sequential convergence (4.13) and the Łojasiewicz-Simon inequality, by using the classical argument in Jendoubi [14], we can show that after a certain time, the trajectory $\phi(t)$ will fall into a certain small neighborhood of $\phi_{\infty}$ and stay there for all time.

Proposition 4.7. There is a $t_{0}>0$, such that $\left\|\phi(t)-\phi_{\infty}\right\|_{H^{1}}<\beta$, for all $t \geq t_{0}$.

Thus, for all $t \geq t_{0}, \phi(t)$ fulfills the condition in Lemma 4.6. Since $(1-\xi)>\frac{1}{2}$, then we infer from (4.12) that

$$
\begin{aligned}
\left(\mathcal{E}(t)-a \lambda_{0} E\left(\phi_{\infty}\right)\right)^{1-\xi} & \leq\left(\|u\|^{2}+\zeta\|\nabla \theta\|^{2}+\omega\|\theta\|^{2}+a \lambda_{0}\left|E(\phi)-E\left(\phi_{\infty}\right)\right|\right)^{1-\xi} \\
& \leq C\left(\|u\|^{2}+\|\nabla \theta\|^{2}+\|\theta\|^{2}\right)^{1-\xi}+C\left|E(\phi)-E\left(\phi_{\infty}\right)\right|^{1-\xi} \\
& \leq C\|u\|+C\|\nabla \theta\|+C\left\|\Delta \phi-F^{\prime}(\phi)\right\|,
\end{aligned}
$$

which combined with the energy inequality (2.3) yields that for $t \geq t_{0}$, it holds that

$$
\begin{aligned}
-\frac{d}{d t}\left(\mathcal{E}(t)-\mathcal{E}_{\infty}\right)^{\xi} & =-\xi\left(\mathcal{E}(t)-a \lambda_{0} E\left(\phi_{\infty}\right)\right)^{\xi-1} \frac{d \mathcal{E}}{d t} \\
& \geq C \xi \frac{\|\nabla u\|^{2}+\left\|\Delta \phi-F^{\prime}(\phi)\right\|^{2}+\|\Delta \theta\|^{2}}{\|u\|+\|\nabla \theta\|+\left\|\Delta \phi-F^{\prime}(\phi)\right\|_{H^{-1}}} \\
& \geq C\left(\|\nabla u\|+\left\|\Delta \phi-F^{\prime}(\phi)\right\|+\|\Delta \theta\|\right) .
\end{aligned}
$$

Integrating (4.16) with respect to $t$, using (4.15) and the fact $\mathcal{E}(t)>\mathcal{E}_{\infty}$, we have

$$
\begin{aligned}
\int_{t_{0}}^{\infty}\left\|\phi_{t}(t)\right\| d t & \leq C \int_{t_{0}}^{\infty}\left(\|\nabla u\|+\left\|\Delta \phi-F^{\prime}(\phi)\right\|\right) d t \\
& \leq C\left(\mathcal{E}\left(t_{0}\right)-\mathcal{E}_{\infty}\right)^{\xi}<+\infty
\end{aligned}
$$

which combined with the compactness of $\phi$ in $H^{1}$ yields that $\lim _{t \rightarrow+\infty} \| \phi(t)-$ $\phi_{\infty} \|_{H^{1}}=0$. Furthermore, since

$$
\left\|\Delta \phi-\Delta \phi_{\infty}\right\| \leq\left\|\Delta \phi-\Delta \phi_{\infty}-F^{\prime}(\phi)+F^{\prime}\left(\phi_{\infty}\right)\right\|+\left\|F^{\prime}(\phi)-F^{\prime}\left(\phi_{\infty}\right)\right\|
$$




$$
\leq\left\|\Delta \phi-F^{\prime}(\phi)\right\|+\left\|\phi(t)-\phi_{\infty}\right\|_{H^{1}},
$$

we conclude from (4.10) that

$$
\lim _{t \rightarrow+\infty}\left\|\phi(t)-\phi_{\infty}\right\|_{H^{2}}=0
$$

It remains to prove the convergence rate (4.7). By Lemma 4.6 and (4.16), we obtain that

$$
\frac{d}{d t}\left(\mathcal{E}(t)-\mathcal{E}_{\infty}\right)+C\left(\mathcal{E}(t)-\mathcal{E}_{\infty}\right)^{2(1-\xi)} \leq 0, \quad \forall t \geq t_{0},
$$

which implies the decay rate for the total energy $\mathcal{E}$

$$
0 \leq \mathcal{E}(t)-\mathcal{E}_{\infty} \leq C(1+t)^{-\frac{1}{1-2 \xi}}, \quad \forall t \geq t_{0} .
$$

Integrating (4.16) on $(t,+\infty)$, where $t \geq t_{0}$, it follows from (4.15) that

$$
\int_{t}^{+\infty}\left\|\phi_{t}(\tau)\right\| d \tau \leq C\left(\mathcal{E}(t)-\mathcal{E}_{\infty}\right)^{\xi} \leq C(1+t)^{-\frac{\xi}{1-2 \xi}} .
$$

Adjusting the constant $C$ properly, we get

$$
\left\|\phi(t)-\phi_{\infty}\right\| \leq C(1+t)^{-\frac{\xi}{1-2 \xi}}, \quad \forall t \geq 0 .
$$

Higher-order estimates on the convergence rate can be achieved by constructing proper differential inequalities via that energy method. It is clear that for the asymptotic limit $\left(\mathbf{0}, \phi_{\infty}, 0\right)$, the system $(1.8)-(1.11)$ is reduced to

$$
\begin{aligned}
\nabla P_{\infty}+\frac{1}{2} \nabla\left(\left|\nabla \phi_{\infty}\right|^{2}\right) & =-\nabla \phi_{\infty} \cdot \Delta \phi_{\infty}, \\
-\Delta \phi_{\infty}+F^{\prime}\left(\phi_{\infty}\right) & =0, \text { with }\left.\phi_{\infty}\right|_{\Gamma}=-1 .
\end{aligned}
$$

Denote $\varphi=\phi-\phi_{\infty}$. Then $(u, \varphi, \theta)$ satisfies

$$
\begin{aligned}
& u_{t}+u \cdot \nabla u+\nabla \tilde{p}-\nu \Delta u \\
= & -a \lambda_{0}\left(\Delta \varphi \nabla \phi+\Delta \phi_{\infty} \nabla \varphi\right)+b \lambda_{0} \nabla \cdot(\theta \nabla \phi \otimes \nabla \phi)+\alpha \theta g \mathbf{j}, \\
& \nabla \cdot u=0, \\
& \varphi_{t}+u \cdot \nabla \phi=\gamma \Delta \varphi-\gamma\left(F^{\prime}(\phi)-F^{\prime}\left(\phi_{\infty}\right)\right), \\
& \theta_{t}+u \cdot \nabla \theta=k \Delta \theta,
\end{aligned}
$$

where we absorb all those gradient terms into the modified pressure $\tilde{p}$.

Multiplying (4.21) by $u,(4.23)$ by $a \lambda_{0}\left(-\Delta \varphi+\left(F^{\prime}(\phi)-F^{\prime}\left(\phi_{\infty}\right)\right)\right)+\varphi$, and (4.24) by $-\Delta \theta$, respectively, integrating over $\Omega$ and adding them together, we have

$$
\begin{aligned}
& \frac{1}{2} \frac{d}{d t} \mathcal{Y}(t)+\nu\|\nabla u\|^{2}+a \lambda_{0} \gamma\left\|\Delta \phi-F^{\prime}(\phi)\right\|^{2}+\gamma\|\nabla \varphi\|^{2}+k\|\Delta \theta\|^{2} \\
&=-b \lambda_{0} \int_{\Omega} \theta \nabla_{i} \phi \nabla_{j} \phi \nabla_{j} u_{i} d x+\alpha g(\theta \mathbf{j}, u)+(u \cdot \nabla \theta, \Delta \theta) \\
&-(u \cdot \nabla \phi, \varphi)-\gamma\left(F^{\prime}(\phi)-F^{\prime}\left(\phi_{\infty}\right), \varphi\right)
\end{aligned}
$$




$$
:=\sum_{m=1}^{5} R_{m},
$$

where

$$
\begin{aligned}
\mathcal{Y}(t)=\|u\|^{2} & +a \lambda_{0}\|\nabla \varphi\|^{2}+2 a \lambda_{0} \int_{\Omega}\left[F(\phi)-F\left(\phi_{\infty}\right)-F^{\prime}\left(\phi_{\infty}\right) \varphi\right] d x \\
& +\|\varphi\|^{2}+\|\nabla \theta\|^{2} .
\end{aligned}
$$

In the derivation of (4.25), we have used (4.19), (4.20), and the following fact

$$
\begin{aligned}
& \int_{\Omega}\left(\Delta \varphi \nabla \phi+\Delta \phi_{\infty} \nabla \varphi\right) \cdot u d x+\int_{\Omega} u \cdot \nabla \phi\left[-\Delta \varphi+\left(F^{\prime}(\phi)-F^{\prime}\left(\phi_{\infty}\right)\right)\right] d x \\
= & \int_{\Omega}\left(\Delta \phi_{\infty}-F^{\prime}\left(\phi_{\infty}\right)\right) \nabla \phi \cdot u d x+\int_{\Omega} u \cdot \nabla F(\phi) d x-\int_{\Omega} \Delta \phi_{\infty} \nabla \phi_{\infty} \cdot u d x \\
= & 0 .
\end{aligned}
$$

Since we are now dealing with global strong solutions that are uniformly bounded in $\mathbf{V} \times H^{2} \times H^{2}$, it follows that

$$
\begin{aligned}
R_{1} & \leq\|\theta\|_{L^{\infty}}\|\nabla u\|\|\nabla \phi\|_{\mathbf{L}^{4}}^{2} \leq C\|\Delta \theta\|^{\frac{3}{4}}\|\theta\|^{\frac{1}{4}}\|\nabla u\|\|\phi\|_{H^{2}}^{2} \\
& \leq \frac{\nu}{12}\|\nabla u\|^{2}+\frac{k}{4}\|\Delta \theta\|^{2}+C\|\theta\|^{2}, \\
R_{2} & \leq\left|\alpha\|g \mid\| \theta\|\| u\left\|\leq \frac{\nu}{12}\right\| \nabla u\left\|^{2}+C\right\| \theta \|^{2},\right. \\
R_{3} & \leq \frac{k}{8}\|\Delta \theta\|^{2}+C\|u\|_{\mathbf{L}^{6}}^{2}\|\nabla \theta\|_{\mathbf{L}^{3}}^{2} \leq \frac{k}{8}\|\Delta \theta\|^{2}+C\|\theta\|^{\frac{1}{2}}\|\Delta \theta\|^{\frac{3}{2}} \\
& \leq \frac{k}{4}\|\Delta \theta\|^{2}+C\|\theta\|^{2}, \\
R_{4}+R_{5} & \leq\|u\|_{\mathbf{L}^{6}}\|\nabla \phi\|_{\mathbf{L}^{3}}\|\varphi\|+C\|\varphi\|^{2} \leq \frac{\nu}{12}\|\nabla u\|^{2}+C\|\varphi\|^{2} .
\end{aligned}
$$

From the definition of $F(\phi)$, we have $\left|\int_{\Omega}\left[F(\phi)-F\left(\phi_{\infty}\right)-F^{\prime}\left(\phi_{\infty}\right) \varphi\right] d x\right| \leq C_{1}\|\varphi\|^{2}$. Combined with the definition of $\mathcal{Y}(t)$ in $(4.26)$, it yields that

$$
\mathcal{Y}(t)+C_{1}\|\varphi\|^{2} \geq C\left(\|u\|^{2}+\|\varphi\|_{H^{1}}^{2}+\|\nabla \theta\|^{2}\right) .
$$

It follows from $(4.25),(4.27)$, and the estimates on $R_{m}(m=1, \ldots, 5)$ that

$$
\frac{d}{d t} \mathcal{Y}(t)+C_{2} \mathcal{Y}(t)+C_{3} \mathcal{A}_{i}(t) \leq C\left(\|\varphi\|^{2}+\|\theta\|^{2}\right), \quad i=1,2 .
$$

Recalling Lemma $3.5(n=2)$ or Lemma $3.7(n=3)$, we have

$$
\frac{d}{d t} \mathcal{A}_{i}(t) \leq C_{4} \mathcal{A}_{i}(t)
$$

Multiplying (4.29) with $\eta=\frac{C_{3}}{2 C_{4}}$, and adding the resultant to (4.28), we get from (4.8) and (4.18) that

$$
\frac{d}{d t}\left[\mathcal{Y}(t)+\eta \mathcal{A}_{i}(t)\right]+C^{\prime}\left[\mathcal{Y}(t)+\eta \mathcal{A}_{i}(t)\right] \leq C\left(\|\varphi\|^{2}+\|\theta\|^{2}\right), \quad \forall t \geq 0
$$


Consequently,

$$
\begin{aligned}
& \mathcal{Y}(t)+\eta \mathcal{A}_{i}(t) \\
\leq & C e^{-C^{\prime} t}+C e^{-C^{\prime} t}\left(\int_{0}^{\frac{t}{2}} e^{C^{\prime} \tau}(1+\tau)^{-\frac{2 \theta}{1-2 \theta}} d \tau+\int_{\frac{t}{2}}^{t} e^{C^{\prime} \tau}(1+\tau)^{-\frac{2 \theta}{1-2 \theta}} d \tau\right) \\
= & C e^{-C^{\prime} t}+C e^{-C^{\prime} t} \int_{0}^{\frac{t}{2}} e^{C^{\prime} \tau}(1+\tau)^{-\frac{2 \theta}{1-2 \theta}} d \tau \\
& +C e^{-C^{\prime} t}\left[\left.\frac{e^{C^{\prime} \tau}}{C^{\prime}}(1+\tau)^{-\frac{2 \theta}{1-2 \theta}}\right|_{\tau=\frac{t}{2}} ^{\tau=t}+\frac{2 \theta}{C^{\prime}(1-2 \theta)} \int_{0}^{t} e^{C^{\prime} \tau}(1+\tau)^{-\frac{1}{1-2 \theta}} d \tau\right] \\
\leq & C e^{-C^{\prime} t}+C e^{-C^{\prime} t}\left(e^{\frac{C^{\prime} t}{2}} \int_{0}^{\frac{t}{2}}(1+\tau)^{-\frac{2 \theta}{1-2 \theta}} d \tau+(1+t)^{-\frac{2 \theta}{1-2 \theta}} e^{C^{\prime} t}\right) \\
\leq & C(1+t)^{-\frac{2 \xi}{1-2 \xi}}, \quad \forall t \geq 0 .
\end{aligned}
$$

Then our conclusion (4.7) follows from (4.30), the definitions of $\mathcal{A}_{i}(t), \mathcal{Y}(t)$, and (4.17). The exponential decay of $\theta$ (see (4.8)) easily follows from (2.6). The proof of Theorem 4.3 is complete.

4.2. Stability of energy minimizers. We have shown that any global weak (or strong) solution of problem (1.8)-(1.15) will converge to a steady state as time goes to infinity (without smallness restrictions on the initial data). However, it is not clear to which equilibrium it will converge, since the set of equilibria may be a continuum (for general Dirichlet boundary data of the phase function). This is different from the classical concept of stability in the literature. Below we shall show that if $u_{0}$ and $\theta_{0}$ are close to zero and $\phi_{0}$ is near a certain local minimizer of the elastic energy $E(\phi)$, problem (1.8)-(1.15) admits a unique global strong solution. Moreover, the energy minimizer is Lyapunov stable. As in Remark 4.1, the results and their proofs in this subsection actually hold for general Dirichlet boundary conditions for $\phi$, not only the special case (1.14).

Definition 4.8. The function $\phi^{*} \in \mathcal{K}:=\left\{\phi \in H^{1}(\Omega):\left.\phi\right|_{\Gamma}=-1\right\}$ is called a local minimizer of $E(\phi)$ if there exists $\sigma>0$, such that for any $\phi \in \mathcal{K}$ satisfying $\left\|\phi-\phi^{*}\right\|_{H^{1}} \leq \sigma$, it holds that $E(\phi) \geq E\left(\phi^{*}\right)$.

REMARK 4.2. It is easy to verify that any local minimizer of $E(\phi)$ is a critical point of $E(\phi)$ and satisfies the elliptic boundary value problem (4.11).

TheOREM 4.9. Suppose $n=3$ and $(2.2)$ is satisfied. Let $\phi^{*} \in H^{2}(\Omega) \cap \mathcal{K}$ be a local minimizer of $E(\phi)$. For arbitrary $r>0$, we consider the set

$$
\begin{gathered}
\mathcal{B}_{r}=\left\{(u, \phi, \theta) \in \mathbf{V} \times\left(H^{2}(\Omega) \cap \mathcal{K}\right) \times\left(H^{2}(\Omega) \cap H_{0}^{1}(\Omega)\right)\right\}: \\
\left.\|u\|_{\mathbf{H}^{1}} \leq r,\left\|\phi-\phi^{*}\right\|_{H^{2}} \leq r,\|\theta\|_{H^{2}} \leq r\right\} .
\end{gathered}
$$

Then there exist positive constants $\sigma_{1}, \sigma_{2}, \sigma_{3}$ depending on $r, \Omega, \sigma, \nu^{*}, \phi^{*}$, and coefficients of the system, such that for any initial data $\left(u_{0}, \phi_{0}, \theta_{0}\right) \in \mathcal{B}_{r}$ satisfying

$$
\left\|u_{0}\right\| \leq \sigma_{1},\left\|\phi_{0}-\phi^{*}\right\|_{H^{1}} \leq \sigma_{2},\left\|\theta_{0}\right\|_{H^{1}} \leq \sigma_{3}
$$

we have 
(i) the problem (1.8)-(1.15) admits a unique global strong solution $(u, \phi, \theta)$;

(ii) the energy minimizer $\phi^{*}$ is Lyapunov stable;

(iii) the global strong solution has the same long-time behavior as in Theorem 4.3. Although the limit function $\phi_{\infty}$ may differ from the minimizer $\phi^{*}$, the total energy $\mathcal{E}(t)$ will converge to the same energy level of $\phi^{*} ;$

$$
\lim _{t \rightarrow+\infty} \mathcal{E}(t)=a \lambda_{0} E\left(\phi_{\infty}\right)=a \lambda_{0} E\left(\phi^{*}\right)
$$

Moreover, if $\phi^{*}$ is an isolated local minimizer, then $\phi_{\infty}=\phi^{*}$ and thus $\phi^{*}$ is asymptotically stable.

Proof. By Proposition 4.1, in order to prove the existence of global strong solutions, we only have to verify that

$$
\mathcal{E}(t)-\mathcal{E}(0) \geq-\varepsilon_{0}, \quad \forall t \in[0,+\infty)
$$

where $\varepsilon_{0}$ is defined as in (4.4). We know from the argument in Proposition 4.1 that there exists $t_{0}=\frac{3}{4} T_{\max }$ and that $\mathcal{A}_{2}(t)$ is uniformly bounded on $\left[0, t_{0}\right]$ by a constant depending on $r, \phi^{*}, \Omega$, and coefficients of the system. Since $\mathcal{A}_{2}(t)$ is bounded on $\left[0, t_{0}\right]$, it holds that

$$
\begin{aligned}
& \mathcal{E}(t)-\mathcal{E}(0) \\
= & \frac{1}{2}\|u(t)\|^{2}-\frac{1}{2}\left\|u_{0}\right\|^{2}+a \lambda_{0}\left(E(\phi(t))-E\left(\phi_{0}\right)\right)+\frac{\zeta}{2}\|\nabla \theta(t)\|^{2} \\
& +\frac{\omega}{2}\|\theta(t)\|^{2}-\frac{\zeta}{2}\left\|\nabla \theta_{0}\right\|^{2}-\frac{\omega}{2}\left\|\theta_{0}\right\|^{2} \\
\geq & -\frac{1}{2}\left\|u_{0}\right\|^{2}+a \lambda_{0}\left(E(\phi(t))-E\left(\phi^{*}\right)+E\left(\phi^{*}\right)-E\left(\phi_{0}\right)\right) \\
& -\frac{\zeta}{2}\left\|\nabla \theta_{0}\right\|^{2}-\frac{\omega}{2}\left\|\theta_{0}\right\|^{2} \\
\geq & -\frac{1}{2}\left\|u_{0}\right\|^{2}-\frac{\zeta}{2}\left\|\nabla \theta_{0}\right\|^{2}-\frac{\omega}{2}\left\|\theta_{0}\right\|^{2}-C_{1}\left\|\phi_{0}-\phi^{*}\right\|_{H^{1}} \\
& +a \lambda_{0}\left(E(\phi(t))-E\left(\phi^{*}\right)\right),
\end{aligned}
$$

where $\zeta$ and $\omega$ are as in Proposition 2.5 and $C_{1}$ depends on $r, \phi^{*}, \Omega$, and coefficients of the system. We assume that $\sigma_{m}(m=1,2,3)$ are sufficiently small such that

$$
\frac{1}{2} \sigma_{1}^{2}+\frac{1}{2} \max \{\zeta, \omega\} \sigma_{3}^{2}+C_{1} \sigma_{2} \leq \varepsilon_{0}
$$

If we can ensure that

$$
E(\phi(t))-E\left(\phi^{*}\right) \geq 0, \quad \forall t \in\left[0, t_{0}\right]
$$

then we have

$$
\mathcal{E}(t)-\mathcal{E}(0) \geq-\varepsilon_{0}, \quad \forall t \in\left[0, t_{0}\right] .
$$

This enables us to apply the argument in the proof for Proposition 4.1 to extend the local strong solution from $\left[0, t_{0}\right]$ to $\left[0, t_{0}+\frac{2 t_{0}}{3}\right]=\left[0, \frac{5 t_{0}}{3}\right]$. 
By Definition 4.8, (4.36) can be reduced to the following condition:

$$
\left\|\phi(t)-\phi^{*}\right\|_{H^{1}}<\min \{\sigma, \beta\}:=\delta, \quad \forall t \in\left[0, t_{0}\right],
$$

where $\beta>0$ is the constant depending on $\phi^{*}$ given in Lemma 4.6 (taking $\psi=\phi^{*}$ therein, we note that $\phi^{*}$ is a critical point of $\left.E(\phi)\right)$. We shall show that one can choose a smaller $\sigma_{2}$ satisfying

$$
\sigma_{2} \leq \frac{1}{4} \delta
$$

such that (4.38) holds. This can be done via the Łojasiewicz-Simon approach by a contradiction argument (cf. Wu et al. [32]). If (4.38) is not true, then since $\phi \in C\left(\left[0, t_{0}\right] ; H_{0}^{1}\right)$, there exists a minimal time $T_{0} \in\left(0, t_{0}\right]$, such that $\left\|\phi\left(T_{0}\right)-\phi^{*}\right\|_{H^{1}}=\delta$. We observe that $\mathcal{E}(t) \geq a \lambda_{0} E\left(\phi^{*}\right)$ for any $t \in\left[0, T_{0}\right]$. If for some $T \leq T_{0}, \mathcal{E}(T)=$ $a \lambda_{0} E\left(\phi^{*}\right)$, then we deduce from the definition of the local minimizer and the basic energy inequality (2.3) that for $t \geq T, \mathcal{E}(t)$ cannot drop and will remain $a \lambda_{0} E\left(\phi^{*}\right)$. Thus, $\nabla u=\Delta \phi-F^{\prime}(\phi)=\Delta \theta \equiv 0$ for all $t \geq T$ and the evolution becomes stationary. The conclusion easily follows. In the following, we just assume $\mathcal{E}(t)>a \lambda_{0} E\left(\phi^{*}\right)$ for $t \in\left[0, T_{0}\right]$. Applying Lemma 4.6 with $\psi=\phi^{*}$, we get (similar to (4.16))

$$
-\frac{d}{d t}\left[\mathcal{E}(t)-a \lambda_{0} E\left(\phi^{*}\right)\right]^{\xi} \geq C\left(\|\nabla u\|+\left\|\Delta \phi-F^{\prime}(\phi)\right\|+\|\Delta \theta\|\right), \quad \forall t \in\left(0, T_{0}\right) .
$$

Then we infer from (4.15) that

$$
\begin{aligned}
\left\|\phi\left(T_{0}\right)-\phi_{0}\right\|_{H^{1}} & \leq C\left\|\phi\left(T_{0}\right)-\phi_{0}\right\|^{\frac{1}{2}}\left\|\phi\left(T_{0}\right)-\phi_{0}\right\|_{H^{2}}^{\frac{1}{2}} \\
& \leq C\left(\int_{0}^{T_{0}}\left\|\phi_{t}(t)\right\| d t\right)^{\frac{1}{2}} \\
& \leq C\left[\mathcal{E}(0)-a \lambda_{0} E\left(\phi^{*}\right)\right]^{\frac{\xi}{2}} \\
& \leq C_{2}\left(\left\|u_{0}\right\|^{2}+\left\|\theta_{0}\right\|_{H^{1}}^{2}+\left\|\phi_{0}-\phi^{*}\right\|_{\mathbf{H}^{1}}\right)^{\frac{\xi}{2}} .
\end{aligned}
$$

Choosing $\sigma_{m}(m=1,2,3)$ satisfying (4.35), (4.39), and

$$
C_{2}\left(\sigma_{1}^{2}+\sigma_{3}^{2}+\sigma_{2}\right)^{\frac{\xi}{2}} \leq \frac{1}{2} \delta
$$

we can see that

$$
\left\|\phi\left(T_{0}\right)-\phi^{*}\right\|_{H^{1}} \leq\left\|\phi\left(T_{0}\right)-\phi_{0}\right\|_{H^{1}}+\left\|\phi_{0}-\phi^{*}\right\|_{H^{1}} \leq \frac{3}{4} \delta<\delta,
$$

which leads to a contradiction with the definition of $T_{0}$. Thus, (4.38) is true and (4.37) holds.

By iteration, we conclude that the local strong solution $(u, \phi, \theta)$ can be extended by a fixed length $\frac{2 t_{0}}{3}$ in each step and it is indeed a global solution with $\mathcal{A}_{2}(t)$ being uniformly bounded. Then by Theorem 4.3 , there exists a critical point $\phi_{\infty}$ of $E(\phi)$, such that the global solution $(u(t), \phi(t), \theta(t))$ satisfies the same long-time behavior (4.5) with convergence rate (4.7). It is easy to see from the above argument that for any $\epsilon>0$, by choosing sufficiently small $\sigma_{m}(m=1,2,3)$, it holds that $\left\|\phi(t)-\phi^{*}\right\|_{H^{1}} \leq$ 
$\epsilon$ for all $t \geq 0$. This implies the Lyapunov stability of the local minimizer $\phi^{*}$. In particular, we have

$$
\left\|\phi_{\infty}-\phi^{*}\right\|_{H^{1}} \leq\left\|\phi(t)-\phi_{\infty}\right\|_{H^{1}}+\left\|\phi(t)-\phi^{*}\right\|_{H^{1}} \leq \min \{\sigma, \beta\} .
$$

Applying the Łojasiewicz-Simon inequality once more with $\psi=\phi^{*}$, we conclude that

$$
\left|E\left(\phi_{\infty}\right)-E\left(\phi^{*}\right)\right|^{1-\xi} \leq\left\|-\Delta \phi_{\infty}+F^{\prime}\left(\phi_{\infty}\right)\right\|=0,
$$

which together with (4.5) yields (4.33). The proof is complete.

Acknowledgment. The authors would like to thank the referees for their very helpful comments and suggestions on an earlier version of this paper. Part of the work was done when Xu was visiting School of Mathematical Sciences at Fudan University, whose hospitality is acknowledged. X. Xu would also like to warmly thank the Center for Nonlinear Analysis at Carnegie Mellon University (NSF Grants No. DMS-0405343 and DMS-0635983), where part of this research was carried out.

\section{REFERENCES}

[1] H. Abels, On a diffuse interface model for two-phase flows of viscous incompressible fluids with matched densities, Arch. Ration. Mech. Anal., 194, 463-506, 2009.

[2] D.M. Anderson, G.B. McFadden, and A.A. Wheeler, Diffuse-interface methods in fluid mechanics, Ann. Rev. Fluid Mech., 30, 139-165, 1998.

[3] R. Borcia and M. Bestehorn, Phase-field model for Marangoni convection in liquid-gas systems with a deformable interface, Phys. Rev. E, 67, 066307, 2003.

[4] R. Borcia and M. Bestehorn, Phase-field simulations for drops and bubbles, Phys. Rev. E, 75, 056309, 2007.

[5] F. Boyer, Mathematical study of multi-phase flow under shear through order parameter formulation, Asymptot. Anal., 20(2), 175-212, 1999.

[6] J.W. Cahn and J.E. Hillard, Free energy of a nonuniform system. I. Interfacial free energy, J. Chem. Phys., 28, 258-267, 1958

[7] B. Climent-Ezquerra, F. Guillén-González, and M. Jesus Moreno-Iraberte, Regularity and timeperiodicity for a nematic liquid crystal model, Nonlinear Anal., 71, 539-549, 2009.

[8] J. Feng, C. Liu, J. Shen, and P. Yue, An energetic variational formulation with phase field methods for interfacial dynamics of complex fluids: Advantages and challenges, in Modeling of Soft Matter, IMA Volumes in Mathematics and its Applications, M.-C. T. Calderer and E. Terentjev (Eds.), Springer, New York, 141, 1-26, 2005.

[9] C. Gal and M. Grasselli, Longtime behavior for a model of homogeneous incompressible twophase flows, Discrete Conti. Dyna. Sys., 28(1), 1-39, 2010.

[10] C. Gal and M. Grasselli, Asymptotic behavior of a Cahn-Hilliard-Navier-Stokes system in 2D, Ann. Inst. H. Poincaré Anal. Non Linéaire, 27(1), 401-436, 2010.

[11] C. Gal and M. Grasselli, Trajectory attractors for binary fluid mixtures in 3D, Chinese Ann. Math. Ser. B, 31, 655-678, 2010.

[12] A. Haraux, Systèmes Dynamiques Dissipatifs et Applications, Masson, Paris, 1991.

[13] A. Haraux and M.A. Jendoubi, Convergence of bounded weak solutions of the wave equation with dissipation and analytic nonlinearity, Calc. Var. PDEs, 9, 95-124, 1999.

[14] M.A. Jendoubi, A simple unified approach to some convergence theorem of L. Simon, J. Func. Anal., 153, 187-202, 1998.

[15] D. Johnson and R. Narayanan, A tutorial on the Rayleigh-Marangoni-Benard problem with multiple layers and side wall effects, Chaos, 9(1), 124-140, 1999.

[16] B. Khouider and E.-S. Titi, An inviscid regularization for the surface quasi-geostrophic equation, Commun. Pure Appl. Math., 61(10), 1331-1346, 2008.

[17] F.-H. Lin and C. Liu, Nonparabolic dissipative system modeling the flow of liquid crystals, Commun. Pure Appl. Math., 48(5), 501-537, 1995.

[18] C. Liu and J. Shen, A phase field model for the mixture of two incompressible fluids and its approximation by a Fourier-spectral method, Physica D, 179, 211-228, 2003. 
[19] C. Liu, J. Shen, J. Feng, and P. Yue, Variational approach in two-phase flows of complex fluids: Transport and induced elastic stress, in Mathematical Models and Methods in Phase Transitions, A. Miranville, Nova Publishers (Eds.), New York, 259-278, 2005.

[20] C. Liu and N. Walkington, An Eulerian description of fluids containing visco-elastic particles, Arch. Rational Mech. Anal., 159, 229-252, 2001.

[21] J. Lowengrub and L. Truskinovsky, Quasi-incompressible Cahn-Hilliard fluids and topological transitions, R. Soc. Lond. Proc. Ser. A Math. Phys. Eng. Sci., 454, 2617-2654, 1998.

[22] C.G.M. Marangoni, Ueber die Ausbreitung der Tropfen einer Flussigkeit auf der Oberflache einer anderen, Ann. Phys. Chem. (Poggendorff), 143(7), 337-354, 1871.

[23] M.A. Mendes-Tatsis and D. Agble, The effect of surfactants on Marangoni convection in the isobutanol/water system, J. Non-Equilib. Thermodyn., 25, 239-249, 2000.

[24] J. Serrin, On the interior of weak solutions of Navier-Stokes equations, Arch. Ration. Mech. Anal., 9, 187-195, 1962.

[25] L. Simon, Asymptotics for a class of nonlinear evolution equation with applications to geometric problems, Ann. Math., 118, 525-571, 1983.

[26] C.V. Sternling and E. Scriven, Interfacial turbulence: Hydrodynamic instability and the marangoni effect, AIChE Journal, 5, 514-523, 1999.

[27] P. Sun, C. Liu, and J. Xu, Phase-field model of thermo-induced Marangoni effects in the mixtures and its numerical similations with mixed finite element method, Commun. Comput. Phys., 6(5), 1095-1117, 2009.

[28] R. Temam, Infinite Dimensional Dynamical Systems in Mechanics and Physics, Springer, New York, 1997.

[29] R. Temam, Navier-Stokes Equations: Theory and Numerical Analysis, AMS, 2001.

[30] J. Thompson, On certain curious motions observable at the surfaces of wine and other alcoholic liquors, Phil. Mag., 10, 330-333, 1855.

[31] H. Wu, X. Xu, and C. Liu, Asymptotic behavior for a nematic liquid crystal model with different kinematic transport properties, Calc. Var. Part. Diff. Equ., 45(3\&4), 319-345, 2012.

[32] H. Wu, X. Xu, and C. Liu, On the general Ericksen-Leslie system: Parodi's relation, wellposedness and stability, Arch. Rational Mech. Anal., DOI: 10.1007/s00205-012-0588-2, 2012, in press.

[33] P. Yue, J. Feng, C. Liu, and J. Shen, A diffuse-interface method for simulating two-phase flows of complex fluids, J. Fluid Mech., 515, 293-317, 2004.

[34] P. Yue, J. Feng, C. Liu, and J. Shen, Interfacial forces and Marangoni flow on a nematic drop retracting in an isotropic fluid, J. Colloid and Interface Sci., 290, 281-288, 2005.

[35] L.-Y. Zhao, H. Wu, and H.-Y. Huang, Convergence to equilibrium for a phase-field model for the mixture of two viscous incompressible fluids, Commun. Math. Sci., 7, 939-962, 2009.

[36] S. Zheng, Nonlinear Evolution Equations, Pitman series Monographs and Survey in Pure and Applied Mathematics, Chapman \& Hall/CRC, Boca Raton (Eds.), Florida, 133, 2004. 